\title{
BMJ Open Systematic review on the instruments used for measuring the association of the level of multimorbidity and clinically important outcomes
}

\author{
Eng Sing Lee (D) , ${ }^{1,2}$ Hui Li Koh, ${ }^{1}$ Elaine Qiao-Ying Ho, ${ }^{3}$ Sok Huang Teo, ${ }^{1}$ \\ Fang Yan Wong, ${ }^{1}$ Bridget L Ryan, ${ }^{4,5}$ Martin Fortin, ${ }^{6}$ Moira Stewart ${ }^{5}$
}

To cite: Lee ES, Koh HL, Ho EQ-Y, et al. Systematic review on the instruments used for measuring the association of the level of multimorbidity and clinically important outcomes. BMJ Open 2021;11:e041219. doi:10.1136/ bmjopen-2020-041219

- Prepublication history and additional supplemental material for this paper are available online. To view these files, please visit the journal online (http://dx.doi.org/10.1136/ bmjopen-2020-041219).

Received 02 June 2020 Revised 13 April 2021 Accepted 19 April 2021
Check for updates

(C) Author(s) (or their employer(s)) 2021. Re-use permitted under CC BY-NC. No commercial re-use. See rights and permissions. Published by BMJ.

For numbered affiliations see end of article.

Correspondence to

Dr Eng Sing Lee;

Eng_Sing_LEE@nhgp.com.sg

\section{ABSTRACT}

Objectives There are multiple instruments for measuring multimorbidity. The main objective of this systematic review was to provide a list of instruments that are suitable for use in studies aiming to measure the association of a specific outcome with different levels of multimorbidity as the main independent variable in community-dwelling individuals. The secondary objective was to provide details of the requirements, strengths and limitations of these instruments, and the chosen outcomes.

Methods We conducted the review according to the Preferred Reporting Items for Systematic Reviews and Meta-Analyses guidelines (PROSPERO registration number: CRD42018105297). We searched MEDLINE, Embase and CINAHL electronic databases published in English and manually searched the Journal of Comorbidity between 1 January 2010 and 23 0ctober 2020 inclusive. Studies also had to select adult patients from primary care or general population and had at least one specified outcome variable. Two authors screened the titles, abstracts and full texts independently. Disagreements were resolved with a third author. The modified Newcastle-Ottawa Scale was used for quality assessment.

Results Ninety-six studies were identified, with 69 of them rated to have a low risk of bias. In total, 33 unique instruments were described. Disease Count and weighted indices like Charlson Comorbidity Index were commonly used. Other approaches included pharmaceutical-based instruments. Disease Count was the common instrument used for measuring all three essential core outcomes of multimorbidity research: mortality, mental health and quality of life. There was a rise in the development of novel weighted indices by using prognostic models. The data obtained for measuring multimorbidity were from sources including medical records, patient self-reports and large administrative databases.

Conclusions We listed the details of 33 instruments for measuring the level of multimorbidity as a resource for investigators interested in the measurement of multimorbidity for its association with or prediction of a specific outcome.

\section{BACKGROUND}

Multimorbidity is defined as the co-occurrence of two or more chronic medical
Strengths and limitations of this study

- This review builds on Huntley et al's 2012 review article and provides an updated, comprehensive list of instruments that measure levels of multimorbidity in community-dwelling individuals.

- A thorough literature search of three major electronic databases was conducted with the involvement of a health science librarian.

- The review is reported based on Preferred Reporting Items for Systematic Reviews and Meta-Analyses guidelines.

This review excluded non-English language articles and grey literature.

conditions in an individual. ${ }^{1}$ It is a growing public health challenge and accounts for most of the expenditures in the healthcare system. ${ }^{2}$ The complex interactions of several coexisting diseases have profound implications on individuals ${ }^{34}$ and their healthcare providers. ${ }^{56}$

There are multiple instruments for measuring multimorbidity and many of them do not usually specify the severity of individual conditions. ${ }^{7}$ No gold standard multimorbidity measurement instrument exists and there is also no agreed categorisation of the available instruments. Sarfati ${ }^{89}$ classified the various measurement instruments into four broad approaches. They are as follows: (1) by simple counts of individual conditions (ie, Disease Count), (2) by organ or systembased approaches, (3) by weighting conditions and combining them into indices and (4) by other miscellaneous approaches. Most of these measurements are used to measure the prevalence or patterns of multimorbidity. However, they can also be used to predict an outcome or to evaluate an intervention for a desired outcome. A set of core outcomes of multimorbidity (COSmm) was proposed 
after consulting a panel of international experts in multimorbidity intervention studies using a Delphi process. ${ }^{10}$ Core outcome sets represent the minimum that should be measured and reported in all clinical trials of multimorbidity. ${ }^{11}$

Huntley et $a l^{12}$ published a systematic review in 2012 describing the instruments used to measure the morbidity burden in primary care and the general population. They found 17 different instruments from 194 articles. The most widely used instruments and those with the most significant evidence of validity were the Charlson Comorbidity Index (CCI), Disease Count and the Adjusted Clinical Groups (ACG) system. ${ }^{12}$ However, this review was conducted in 2009 and multimorbidity research has increased exponentially since then.

The present review was to build on the review article by Huntley $e t a l^{12}$ in order to provide a current and comprehensive list of instruments that measure levels of multimorbidity for community-dwelling individuals. We used the term 'level of multimorbidity' to refer to the combined effects of multiple conditions on an individual. The main objective of this review was to list instruments for measuring the levels of multimorbidity. We specifically look for studies that measure the association of a clinically important outcome with different levels of multimorbidity as the main independent variable in community-dwelling individuals. Our second objective was to provide details of the requirements, strengths and limitations of these instruments, and the chosen outcomes in the studies so that clinicians and researchers can select or develop instruments that match their needs for predicting a specific outcome.

\section{METHODS}

A protocol for this systematic review (CRD42018105297) was published online on PROSPERO. ${ }^{13}$ We searched MEDLINE, EMBASE, CINAHL and also manually searched the Journal of Comorbidity for potential studies. The medical subject headings and keywords used for the search are shown in online supplemental appendix 1 .

We selected studies that included (1) adult patients from primary care or the general population as the majority of patients with multimorbidity are managed by primary care physicians ${ }^{14}$; (2) at least one specified outcome variable; and (3) published full-text articles from 1 January 2010 to 23 October 2020. Studies were excluded if they (1) selected patients from the hospital or nursing home only or patient data were drawn solely from the hospital or the nursing home; or (2) selected patients with an index condition; or (3) used level of multimorbidity as a covariate and not the main independent variable; or (4) were not written in English. We did not include a specific definition of multimorbidity instrument because, given a lack of consensus in the literature on the use of this term, we wanted to include a diverse range of studies on the above topic.
One reviewer (ESL) conducted a preliminary screen of titles and abstracts to exclude articles that were irrelevant. Abstracts of the remaining articles were screened independently by two reviewers (ESL and EQ-YH) according to the eligibility criteria. Disagreements were resolved through discussion until a consensus was reached. The full-text articles were then retrieved for the agreed list and independently assessed according to the eligibility criteria by the same reviewers. Disagreements were resolved through discussion with a third reviewer (TSH) until a consensus was reached. After agreement on the list of articles, the reference lists of included articles were hand-searched for additional eligible articles. We reported multimorbidity instruments that were described in all selected articles.

The risk of bias of the study design of selected articles was next appraised independently by three reviewers (ESL, EH and TSH) using the modified Newcastle-Ottawa Scale (NOS).${ }^{1516}$ Each article was assessed under the three broad categories: (1) selection, (2) comparability and (3) outcome (online supplemental appendices 2 and 3 ).

We contacted the authors, as needed, for additional information or clarification up to three times spaced 1 week apart. We contacted 25 authors and 19 of them replied. Any disagreements on the risk of bias were resolved among the three reviewers through regular meetings. HLK and FYW were responsible for tracking and updating the final outcome of the risk of bias assessment.

\section{Patient and public involvement}

This research was done without patient involvement. Patients were not invited to comment on the study design and were not consulted to develop patient relevant outcomes or to interpret the results. Patients were not invited to contribute to the writing or editing of this document for readability or accuracy.

\section{RESULTS}

The number of included studies was 96 , of which 69 were assessed to have low risk of bias. A summary of the PRISMA (Preferred Reporting Items for Systematic Reviews and Meta-Analyses) flow chart is depicted in figure 1. Fortyeight studies selected participants from the general population and the other 48 studies selected participants from primary care. Most of the studies in this systematic review were from Europe and North America with very few Asian studies. There were 44 cohort studies, of which 36 were assessed to have low risk of bias, and 52 cross-sectional studies, of which 33 were assessed to have low risk of bias. We found 33 unique instruments from the 96 studies. The instruments were categorised according to Sarfati ${ }^{89}$ into (1) simple counts of individual conditions; (2) organ or system-based approaches; (3) conditions that have been weighted and combined into indices; and (4) other approaches. A total of 150 outcomes were reported from all the studies. No studies were excluded for an outcome that was not deemed to be clinically important. Online 


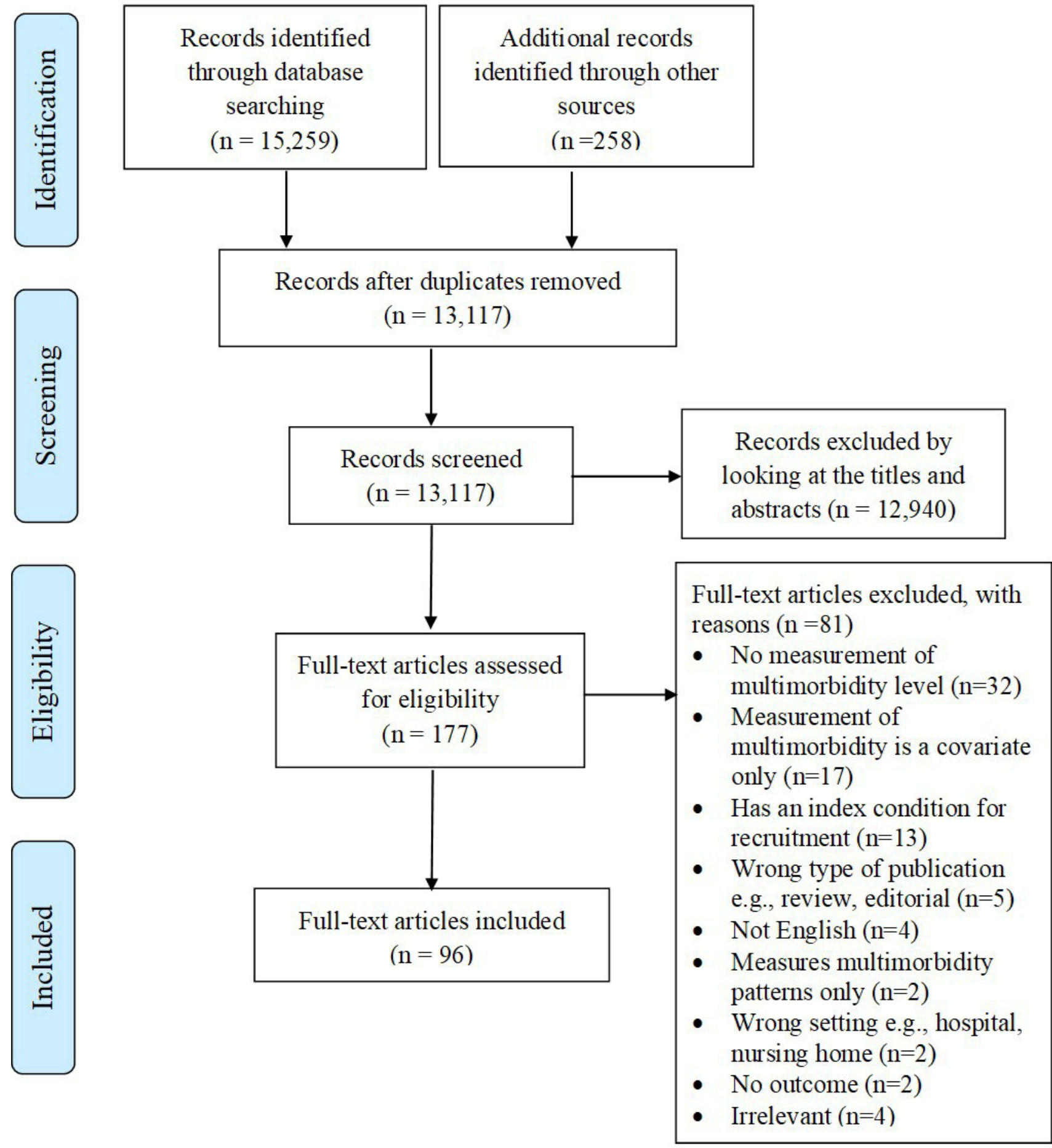

Figure 1 PRISMA (Preferred Reporting Items for Systematic Reviews and Meta-Analyses) flow diagram.

supplemental appendices 4 and 5 summarise the risk of bias assessment of each study. Table 1 provides a summary of the study design, population source, age group, multimorbidity measurements, outcome measures and risk of bias assessment of all the studies.

Table 2 summarises the 33 instruments that were identified from all the studies. Table 3 provides a summary of multimorbidity instruments and their associations with the outcomes measured from all the included studies.

\section{Simple counts of individual conditions}

Disease Count was based on the total number of all the conditions an individual had, usually from a prespecified list of chronic conditions. It was used in 59 out of the 96 studies $(61.5 \%)$. Disease Count was reported to be associated with activity limitations, continuity of care, disability, healthcare cost, healthcare utilisation, medications, mental disorders, mortality, general health, physical function, quality of life and self-rated health (table 3). 
Table 1 Summary of included studies

\begin{tabular}{|c|c|c|c|c|c|c|}
\hline Author (Year) & $\begin{array}{l}\text { Study } \\
\text { design }\end{array}$ & $\begin{array}{l}\text { Population } \\
\text { source }\end{array}$ & Age & $\begin{array}{l}\text { Multimorbidity } \\
\text { measurement }\end{array}$ & Outcomes measured & $\begin{array}{l}\text { Risk of } \\
\text { bias }\end{array}$ \\
\hline $\begin{array}{l}\text { Agborsangaya et al } \\
(2013)^{41}\end{array}$ & CS & GP & $\geq 18$ & DC & HRQoL & Good \\
\hline Bähler et al (2015) ${ }^{42}$ & CS & GP & $\geq 65$ & $\begin{array}{l}\text { DC-ATC classification } \\
\text { system }\end{array}$ & $\begin{array}{l}\text { Total number of } \\
\text { consultations }\end{array}$ & Good \\
\hline Barile et al (2013) ${ }^{43}$ & Cohort & GP & $\geq 65$ & $\mathrm{DC}$ & $\begin{array}{l}\text { ADL limitations, } \\
\text { physically unhealthy } \\
\text { days, mentally } \\
\text { unhealthy days }\end{array}$ & Good \\
\hline Barile et al (2012) & CS & GP & $\geq 65$ & $\mathrm{DC}$ & $\begin{array}{l}\text { Physical HRQoL, } \\
\text { mental HRQoL }\end{array}$ & Good \\
\hline Barnett et al (2012) ${ }^{45}$ & $\mathrm{CS}$ & PC & $\geq 0$ & $\mathrm{DC}$ & $\begin{array}{l}\text { Presence of mental } \\
\text { health disorder }\end{array}$ & Good \\
\hline $\begin{array}{l}\text { Boeckxstaens et al } \\
(2015 b)^{18}\end{array}$ & Cohort & PC & $\geq 80$ & $\mathrm{DC}, \mathrm{mCCl}, \mathrm{CIRS}$ & $\begin{array}{l}\text { Mortality at } 3 \text { years, } \\
\text { hospitalisation at } \\
3 \text { years, functional } \\
\text { decline at } 19 \text { months } \\
\text { (ADL, physical, mental } \\
\text { decline) }\end{array}$ & Fair \\
\hline $\begin{array}{l}\text { Brilleman et al } \\
(2014)^{47}\end{array}$ & Cohort & PC & $\geq 18$ & $\begin{array}{l}\text { QOF count, } \mathrm{CCl} \text {, EDC } \\
\text { count, ACG, RUB }\end{array}$ & $\begin{array}{l}\text { Primary healthcare cost } \\
\text { The EDC performed } \\
\text { best followed by the } \\
\text { QOF and ACG }\end{array}$ & Good \\
\hline $\begin{array}{l}\text { Caballer-Tarazona et } \\
\text { al (2019) }\end{array}$ & CS & GP & $\geq 0$ & CRG & $\begin{array}{l}\text { Expenditure of } \\
\text { integrated healthcare } \\
\text { (hospital, primary } \\
\text { healthcare (PHC) } \\
\text { and pharmaceutical } \\
\text { prescription) }\end{array}$ & Poor \\
\hline
\end{tabular}


Table 1 Continued

\begin{tabular}{llll} 
Author (Year) & $\begin{array}{l}\text { Study } \\
\text { design }\end{array}$ & $\begin{array}{l}\text { Population } \\
\text { source }\end{array}$ & Age \\
\hline Carey et al $(2013)^{50}$ & Cohort & PC & $\geq 60$
\end{tabular}

Multimorbidity measurement

Standard QOF, extended Mortality (1-year QOF, CCI (Khan)

period)
Risk of

bias

Good

The standard QOF

score outperformed

the $\mathrm{CCl}$ (Khan). The

extended QOF score

produced only a

modest improvement

in overall model

performance.

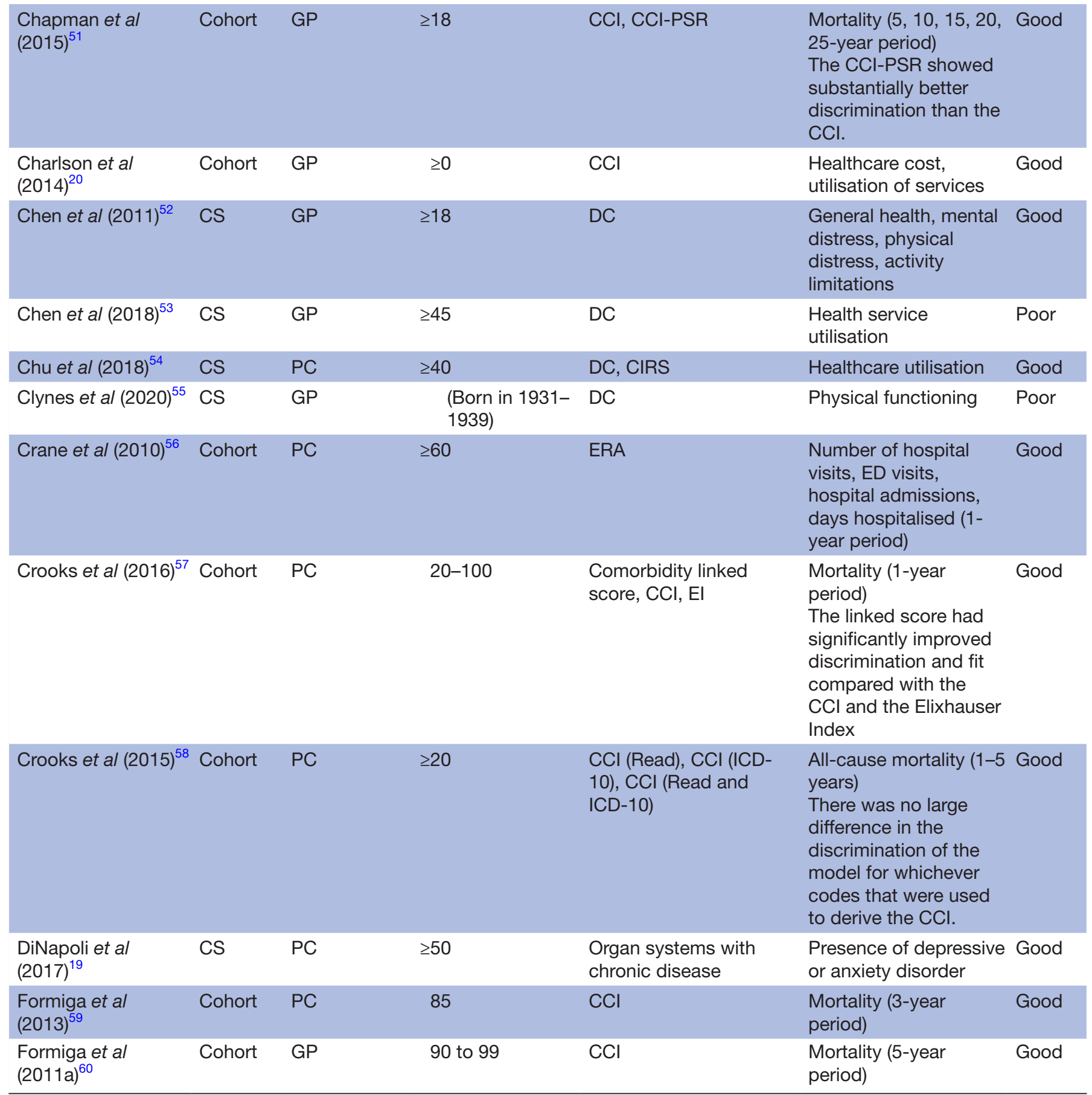

Continued 
Table 1 Continued

\begin{tabular}{|c|c|c|c|c|c|c|}
\hline Author (Year) & $\begin{array}{l}\text { Study } \\
\text { design }\end{array}$ & $\begin{array}{l}\text { Population } \\
\text { source }\end{array}$ & Age & $\begin{array}{l}\text { Multimorbidity } \\
\text { measurement }\end{array}$ & Outcomes measured & $\begin{array}{l}\text { Risk of } \\
\text { bias }\end{array}$ \\
\hline $\begin{array}{l}\text { Formiga et al } \\
(2011 b)^{61}\end{array}$ & CS & PC & 85 & $\mathrm{CCl}$ & Successful ageing & Good \\
\hline $\begin{array}{l}\text { Formiga et al } \\
(2016)^{62}\end{array}$ & Cohort & PC & 85 & $\mathrm{CCl}$ & $\begin{array}{l}\text { Mortality (5-year } \\
\text { period) }\end{array}$ & Good \\
\hline $\begin{array}{l}\text { Fraccaro et al } \\
(2016)^{63}\end{array}$ & Cohort & PC & $\geq 18$ & $\mathrm{CCl}$ (Khan) & $\begin{array}{l}\text { Mortality }(1,5,10 \text {-year } \\
\text { period), mortality ( } 3,6 \text {, } \\
12 \text {-month period) }\end{array}$ & Good \\
\hline Galenkamp et al & CS & GP & $57-98$ & DC & SRH & Good \\
\hline
\end{tabular}

$$
(2011)^{64}
$$

$\begin{array}{lllll}\text { Garin et al (2014) }^{65} & \text { CS } & \text { GP } & \geq 50 & \text { DC } \\ \text { Glynn et al (2011) } & \text { CS } & \text { PC } & >50 & \text { DC }\end{array}$

DC

QOL, disability

Good

Primary care

Good

consultations, hospital

outpatient visits,

hospital admissions,

healthcare cost (all 1-

year period)

\begin{tabular}{|c|c|c|c|c|c|c|}
\hline Gunn et al (2012) ${ }^{67}$ & CS & PC & $18-76$ & DC & $\begin{array}{l}\text { Depressive symptoms } \\
\text { (CES-D score) }\end{array}$ & Fair \\
\hline Haas et al $(2013)^{21}$ & Cohort & PC & $\geq 18$ & $\begin{array}{l}\text { ACG, Minnesota } \\
\text { Healthcare Home } \\
\text { Tiering, HCC, ERA, } \\
\text { CCC, CCI, hybrid model }\end{array}$ & $\begin{array}{l}\text { Hospitalisation, ED } \\
\text { visits, readmission } \\
\text { within } 30 \text { days, } \\
\text { healthcare expenditure } \\
\text { (all 1-year period) } \\
\text { The ACG model } \\
\text { outperformed the } \\
\text { other five models in all } \\
\text { outcomes. }\end{array}$ & Good \\
\hline $\begin{array}{l}\text { Hanmer et al } \\
(2010)^{68}\end{array}$ & CS & GP & 22 to 106 & $\begin{array}{l}\text { Additive model, } \\
\text { minimum model, } \\
\text { multiplicative model }\end{array}$ & Health utility (SF-6D) & Fair \\
\hline Hu et al $(2017)^{69}$ & CS & PC & $\geq 65$ & Age-adjusted CCI & $\begin{array}{l}\text { Frequency of family } \\
\text { physician visits }\end{array}$ & Fair \\
\hline Hwang et al (2015) $)^{23}$ & Cohort & GP & $\geq 0$ & ACE-27, ACE-27 count & $\begin{array}{l}\text { Healthcare expenditure } \\
\text { The model, using year } \\
1 \text { data to determine } \\
\text { if an individual would } \\
\text { be classified into the } \\
\text { persistent high-user } \\
\text { group for the following } \\
3 \text { years, indicates } \\
\text { a very high level of } \\
\text { accuracy in predicting } \\
\text { membership in a high- } \\
\text { user group. }\end{array}$ & Good \\
\hline Isaacs et al (2014) & CS & PC & $18-101$ & $\mathrm{DC}$ & Prescription costs & Poor \\
\hline $\begin{array}{l}\text { Jennings et al } \\
(2015)^{71}\end{array}$ & Cohort & PC & $\geq 75$ & DC & $\begin{array}{l}\text { Count of fall-related } \\
\text { injuries in the } 24 \\
\text { months after the date } \\
\text { of screening }\end{array}$ & Fair \\
\hline Jia et al $(2018)^{72}$ & Cohort & GP & $\geq 65$ & DC & $\begin{array}{l}\text { Quality-adjusted life } \\
\text { years (QALY) }\end{array}$ & Poor \\
\hline $\begin{array}{l}\text { Jia and Lebetkin } \\
(2017)^{73}\end{array}$ & Cohort & GP & $\geq 65$ & DC & $\begin{array}{l}\text { Quality-adjusted life } \\
\text { years (QALY) }\end{array}$ & Poor \\
\hline
\end{tabular}

Continued 
Table 1 Continued

\begin{tabular}{lllll}
\hline Author (Year) & $\begin{array}{l}\text { Study } \\
\text { design }\end{array}$ & $\begin{array}{l}\text { Population } \\
\text { source }\end{array}$ & Age & $\begin{array}{l}\text { Multimorbidity } \\
\text { measurement }\end{array}$ \\
\hline Jindai et al $(2016)^{74}$ & CS & GP & $\geq 65$ & DC
\end{tabular}

Outcomes measured

Risk of

Functional limitations Good

(ADL, IADL, leisure and social activities, lowerextremity mobility, general physical activities)

\begin{tabular}{|c|c|c|c|c|}
\hline Kim et al (2012) ${ }^{75}$ & CS & GP & $\geq 65$ & DC \\
\hline Kojima et al $(2011)^{76}$ & CS & PC & $\geq 65$ & $\mathrm{D}$ \\
\hline $\begin{array}{l}\text { Kristensen et al } \\
(2014)^{77}\end{array}$ & CS & $\mathrm{PC}$ & $>0$ & \\
\hline Lapi et al (2015) ${ }^{78}$ & CS & $\mathrm{PC}$ & $\geq 15$ & \\
\hline
\end{tabular}

Quality of life (EQ5D) Poor

Fall tendency Poor

Fee-for-services Good expenditures

Total mean healthcare Good

cost per year

The HSMI explained

$50.17 \%$ of the variation in costs

$\begin{array}{llccl}\begin{array}{l}\text { Lawson et al } \\ (2013)^{79}\end{array} & \text { CS } & \text { GP } & \geq 20 & \text { DC } \\ {\text { Lemke et al }(2012)^{80}}^{80} & \text { Cohort } & \text { GP } & \geq 0 & \text { CCl, ACG }\end{array}$

Preference-weighted Good HRQoL

Inpatient Good

hospitalisations

ACG-based predictive model was superior to $\mathrm{CCl}$ model.

\begin{tabular}{|c|c|c|c|c|c|c|}
\hline Li et al (2016) $)^{81}$ & CS & GP & $16-68$ & DC & $\begin{array}{l}\text { Health-related quality } \\
\text { of life }\end{array}$ & Poor \\
\hline $\begin{array}{l}\text { Loprinzi et al } \\
(2016)^{82}\end{array}$ & CS & GP & $60-85$ & $\mathrm{DC}$ & Cognitive function & Good \\
\hline $\begin{array}{l}\text { Macinko et al } \\
(2019)^{83}\end{array}$ & CS & GP & $\geq 18$ & $\begin{array}{l}\text { DC (categorical } 2 \text { and } 3 \\
\text { or more) (self-reported) }\end{array}$ & $\begin{array}{l}\text { Primary care } \\
\text { experience (self- } \\
\text { reported) }\end{array}$ & Good \\
\hline $\begin{array}{l}\text { McDaid et al } \\
(2013)^{85}\end{array}$ & CS & GP & $\geq 50$ & DC & Disability, QoL, SRH & Good \\
\hline $\begin{array}{l}\text { Md Yusof et al } \\
(2010)^{86}\end{array}$ & Cohort & GP & $64-85$ & $\mathrm{CCl}$ & Mortality over 7 years & Fair \\
\hline $\begin{array}{l}\text { Muggah et al } \\
(2012)^{89}\end{array}$ & CS & GP & $\geq 20$ & DC & Primary healthcare use & Poor \\
\hline $\begin{array}{l}\text { Mujica-Mota et al } \\
(2015)^{90}\end{array}$ & CS & $\mathrm{PC}$ & $\geq 18$ & $\mathrm{DC}$ & $\begin{array}{l}\text { Health-related quality } \\
\text { of life (EQ5D) }\end{array}$ & Fair \\
\hline $\begin{array}{l}\text { Naessens et al } \\
(2011)^{91}\end{array}$ & CS & GP & $18-64$ & DC & Healthcare cost & Poor \\
\hline $\begin{array}{l}\text { Østergaard and } \\
\text { Foldager }(2011)^{92}\end{array}$ & CS & PC & $\geq 18$ & DC & $\begin{array}{l}\text { Major depressive } \\
\text { episode (measured by } \\
\text { DSQ) }\end{array}$ & Poor \\
\hline
\end{tabular}

Continued 
Table 1 Continued

\begin{tabular}{|c|c|c|c|c|c|c|}
\hline Author (Year) & $\begin{array}{l}\text { Study } \\
\text { design }\end{array}$ & $\begin{array}{l}\text { Population } \\
\text { source }\end{array}$ & Age & $\begin{array}{l}\text { Multimorbidity } \\
\text { measurement }\end{array}$ & Outcomes measured & $\begin{array}{l}\text { Risk of } \\
\text { bias }\end{array}$ \\
\hline $\begin{array}{l}\text { Palladino et al } \\
(2019)^{93}\end{array}$ & CS & GP & $\geq 50$ & DC & $\begin{array}{l}\text { Primary care use, } \\
\text { reduced functional } \\
\text { capacity, self-perceived } \\
\text { health, hospital } \\
\text { admissions, quality of } \\
\text { life }\end{array}$ & Good \\
\hline Pati et al (2019) ${ }^{94}$ & CS & PC & $\geq 18$ & $\begin{array}{l}\text { Severity burden score } \\
\text { ( } 21 \text { conditions) }\end{array}$ & $\begin{array}{l}\text { Health-related quality } \\
\text { of life (SF-12) }\end{array}$ & Good \\
\hline Payne et al (2013) ${ }^{95}$ & Cohort & PC & $\geq 20$ & DC & $\begin{array}{l}\text { Unplanned hospital } \\
\text { admission, potentially } \\
\text { preventable admission } \\
\text { (all 1-year period) }\end{array}$ & Good \\
\hline Payne et al $(2014)^{96}$ & Cohort & PC & $\geq 20$ & $\mathrm{DC}$ & $\begin{array}{l}\text { Unplanned hospital } \\
\text { admissions (1-year } \\
\text { period) }\end{array}$ & Good \\
\hline Payne et al (2020) ${ }^{97}$ & Cohort & PC & $\geq 20$ & $\begin{array}{l}\text { CCI, DC (37 read } \\
\text { codes), Cambridge } \\
\text { Multimorbidity Score }\end{array}$ & $\begin{array}{l}\text { Mortality, unplanned } \\
\text { inpatient hospital } \\
\text { admission, primary } \\
\text { care consultations }\end{array}$ & Good \\
\hline Peters et al (2018) & CS & PC & $18-101$ & DC, DBIS & Quality of life & Fair \\
\hline Quail et al (2011) ${ }^{99}$ & Cohort & GP & $\geq 20$ & $\begin{array}{l}\text { DC, CCI (Quan), } \\
\text { Elixhauser (Quan), } \\
\text { number of different } \\
\text { dispended drugs, CDS }\end{array}$ & $\begin{array}{l}\text { Mortality (1-year } \\
\text { period): Elixhauser } \\
\text { (Quan) performed best } \\
\text { followed by CCl. } \\
\text { One or more } \\
\text { hospitalisations; two or } \\
\text { more hospitalisations: } \\
\text { DC was the best } \\
\text { performing measure }\end{array}$ & Good \\
\hline $\begin{array}{l}\text { Ranstad et al } \\
(2014)^{100}\end{array}$ & CS & GP & $\geq 0$ & RUB & $\begin{array}{l}\text { Registered active } \\
\text { listing in primary care } \\
\text { and all healthcare }\end{array}$ & Good \\
\hline $\begin{array}{l}\text { Reinke et al } \\
(2019)^{101}\end{array}$ & CS & PC & $30-94$ & DC & $\begin{array}{l}\text { Symptom burden } \\
\text { (MSAS-SF), quality of } \\
\text { life (Veterans RAND 12) }\end{array}$ & Good \\
\hline $\begin{array}{l}\text { Renne and Gobbens } \\
(2018)^{102}\end{array}$ & CS & PC & $\geq 70$ & DC & Quality of life & Poor \\
\hline Reyes et al (2014) $)^{103}$ & Cohort & PC (men) & $\geq 65$ & $\mathrm{CCl}$ & Hip fractures & Good \\
\hline Ryu et al $(2015)^{104}$ & CS & PC & $\geq 18$ & $\mathrm{DC}$ & $\begin{array}{l}\text { Deficits of perceived } \\
\text { general health, } \\
\text { depressive symptoms }\end{array}$ & Good \\
\hline $\begin{array}{l}\text { Salisbury et al } \\
(2011)^{105}\end{array}$ & Cohort & PC & $\geq 18$ & QOF count, EDC count & $\begin{array}{l}\text { Primary care } \\
\text { consultation rates, } \\
\text { continuity of care (all } \\
\text { 3-year period) }\end{array}$ & Good \\
\hline Saver et al $(2014)^{106}$ & Cohort & GP & $\geq 65$ & $\begin{array}{l}\mathrm{CCl} \\
\text { (Romano)+Hypertension }\end{array}$ & $\begin{array}{l}\text { Acute ACSH, chronic } \\
\text { ACSH }\end{array}$ & Good \\
\hline
\end{tabular}


Table 1 Continued

\begin{tabular}{|c|c|c|c|c|c|c|}
\hline Author (Year) & $\begin{array}{l}\text { Study } \\
\text { design }\end{array}$ & $\begin{array}{l}\text { Population } \\
\text { source }\end{array}$ & Age & $\begin{array}{l}\text { Multimorbidity } \\
\text { measurement }\end{array}$ & Outcomes measured & $\begin{array}{l}\text { Risk of } \\
\text { bias }\end{array}$ \\
\hline $\begin{array}{l}\text { Shadmi et al } \\
(2011)^{107}\end{array}$ & CS & GP & $\geq 18$ & $\mathrm{ADG}, \mathrm{CCl}$ & $\begin{array}{l}\text { Number of primary } \\
\text { care physician visits, } \\
\text { specialist visits, } \\
\text { hospitalisation } \\
\text { ADG explained } \\
\text { the largest percent } \\
\text { of variance or in } \\
\text { healthcare resource } \\
\text { use }\end{array}$ & Good \\
\hline Sibley et al (2014) ${ }^{108}$ & CS & GP & $\geq 65$ & DC & $\begin{array}{l}\text { Self-reported falls in } \\
\text { the last } 12 \text { months }\end{array}$ & Poor \\
\hline $\begin{array}{l}\text { Stanley and Sarfati } \\
(2017)^{109}\end{array}$ & Cohort & PC & $\geq 18$ & $\begin{array}{l}\text { M3 Index, CCl, } \\
\text { Elixhauser (van } \\
\text { Walraven) }\end{array}$ & $\begin{array}{l}\text { Mortality, overnight } \\
\text { hospitalisation (all 1- } \\
\text { year period) } \\
\text { M3 Index outperformed } \\
\text { both CCI and } \\
\text { Elixhauser (van } \\
\text { Walraven) }\end{array}$ & Good \\
\hline $\begin{array}{l}\text { St John et al } \\
(2014)^{110}\end{array}$ & Cohort & GP & $\geq 65$ & DC (0-36 conditions) & Mortality in 5 years & Good \\
\hline $\begin{array}{l}\text { St John et al } \\
(2019)^{111}\end{array}$ & Cohort & GP & $\geq 65$ & DC & $\begin{array}{l}\text { Functional impairment } \\
\text { in } 5 \text { years }\end{array}$ & Good \\
\hline Streit et al $(2014)^{27}$ & Cohort & PC & $50-80$ & $\mathrm{CCl}, \mathrm{DC}$ & $\begin{array}{l}\text { Quality of } \\
\text { cardiovascular } \\
\text { preventive care, quality } \\
\text { of preventive care }\end{array}$ & Good \\
\hline Tyack et al (2016) ${ }^{115}$ & Cohort & PC & $\geq 18$ & $\mathrm{DC}$ & $\begin{array}{l}\text { Health-related quality } \\
\text { of life }\end{array}$ & Fair \\
\hline $\begin{array}{l}\text { Ubalde-Lopez et al } \\
(2016)^{24}\end{array}$ & CS & GP & $\begin{array}{l}\mathrm{F}(\text { mean): } 35.9 \\
\mathrm{M} \text { (mean): } 37.9\end{array}$ & MDMS & $\begin{array}{l}\text { Sickness absence } \\
\text { episodes taken in last } \\
2 \text { years }\end{array}$ & Good \\
\hline $\begin{array}{l}\text { van den Bussche et } \\
\text { al }(2011)^{116}\end{array}$ & CS & PC & $\geq 65$ & DC & $\begin{array}{l}\text { Frequency of contacts } \\
\text { with physicians, } \\
\text { number of different } \\
\text { ambulatory physicians } \\
\text { contacted (all 1-year } \\
\text { period) }\end{array}$ & Good \\
\hline $\begin{array}{l}\text { van Oostrom et al } \\
(2014)^{117}\end{array}$ & CS & PC & $\geq 55$ & DC & $\begin{array}{l}\text { Number of contacts } \\
\text { with general practice, } \\
\text { medications } \\
\text { prescribed, referrals }\end{array}$ & Good \\
\hline Vos et al $(2013)^{118}$ & CS & PC & $70-74$ & $\mathrm{DC}$ & $\begin{array}{l}\text { Self-rated health (SF- } \\
36)\end{array}$ & Poor \\
\hline
\end{tabular}


Table 1 Continued

\begin{tabular}{|c|c|c|c|c|c|c|}
\hline Author (Year) & $\begin{array}{l}\text { Study } \\
\text { design }\end{array}$ & $\begin{array}{l}\text { Population } \\
\text { source }\end{array}$ & Age & $\begin{array}{l}\text { Multimorbidity } \\
\text { measurement }\end{array}$ & Outcomes measured & $\begin{array}{l}\text { Risk of } \\
\text { bias }\end{array}$ \\
\hline $\begin{array}{l}\text { Wallace et al } \\
(2016 a)^{119}\end{array}$ & Cohort & PC & $\geq 70$ & $\begin{array}{l}\text { Pra tool, modified Pra } \\
\text { tool }\end{array}$ & $\begin{array}{l}\text { Emergency hospital } \\
\text { admission (1-year } \\
\text { period) } \\
\text { Both models } \\
\text { demonstrated poor } \\
\text { model discrimination }\end{array}$ & Good \\
\hline
\end{tabular}

\begin{tabular}{|c|c|c|c|c|c|c|}
\hline Wei et al (2018) $)^{121}$ & CS & GP & $\geq 51$ & $\mathrm{MWI}$ & $\begin{array}{l}\text { Subjective physical } \\
\text { functioning, grip } \\
\text { strength, gait speed, } \\
\text { cognitive performance, } \\
\text { ADL limitations, IADL } \\
\text { limitations }\end{array}$ & Good \\
\hline Wei et al $(2019 a)^{122}$ & Cohort & GP & $\geq 51$ & MWI & $\begin{array}{l}\text { Physical functioning- } \\
\text { SF-36, mortality }\end{array}$ & Good \\
\hline Wei et al $(2020 a)^{124}$ & Cohort & GP & $\geq 51$ & $\mathrm{MWI}$ & Cognitive functioning & Good \\
\hline Wei et al (2020b) $)^{125}$ & Cohort & GP & $\geq 51$ & $\begin{array}{l}\text { MWI-ICD, DC, CCI, } \\
\text { Elixhauser, health- } \\
\text { related quality of life } \\
\text { comorbidity index }\end{array}$ & $\begin{array}{l}\text { Mortality, future } \\
\text { physical functioning }\end{array}$ & Poor \\
\hline $\begin{array}{l}\text { Wikman et al } \\
(2011)^{126}\end{array}$ & CS & GP & $\geq 50$ & DC & $\begin{array}{l}\text { QoL, affective well- } \\
\text { being }\end{array}$ & Good \\
\hline Wister et al (2015) ${ }^{22}$ & CS & GP & $\geq 65$ & $\begin{array}{l}\text { MM additive scale, MM } \\
\text { weighted by HUI3, MM } \\
\text { weighted by ADL scale, } \\
\text { MM weighted by HUI3 } \\
\text { betas }\end{array}$ & $\begin{array}{l}\text { Life satisfaction, } \\
\text { perceived health status }\end{array}$ & Good \\
\hline
\end{tabular}

ACE, Adult Comorbidity Evaluation; ACG, Adjusted Clinical Groups; ACSH, Ambulatory Care Sensitive Hospitalisation; ADG, Aggregated Diagnosis Groups; ADL, Activities of Daily Living; CCC, Chronic Condition Count; CCI, Charlson Comorbidity Index; CCI-PSR, Charlson Comorbidity Index-Psychosocial Risk; CDS, Chronic Disease Score; CIRS, Cumulative Illness Rating Scale; CRG, Clinical Risk Groups; CS, Cross-Sectional; DBIS, Disease Burden Impact Scale; DC, Disease Count (Unweighted); ED, Emergency Department; EDC, Expanded Diagnosis Clusters; El, Elixhauser Index; ERA, Elder Risk Assessment; GP, General Population; HCC, Hierarchical Condition Categories; HRQoL, Health-Related Quality of Life; HSMI, Health Search Morbidity Index; HUI3, Health Utility Index; IADL, Instrumental Activities of Daily Living; ICD-10, International Classification of Diseases, Tenth Revision; mCCl, modified Charlson Comorbidity Index; MDMS, Multidimensional Multimorbidity Score; M3 Index, Multimorbidity Measure Index; MM, Multimorbidity; MWI, Multimorbidity-Weighted Index; PC, Primary Care; Pra tool, Probability of repeated admission risk prediction tool; QOF, Quality and Outcomes Framework; QoL, Quality of Life; RUB, Resource Utilisation Band; RxRisk-V, A Veterans Association adapted pharmacy-based case-mix instrument; SRH, Self-Rated Health. 


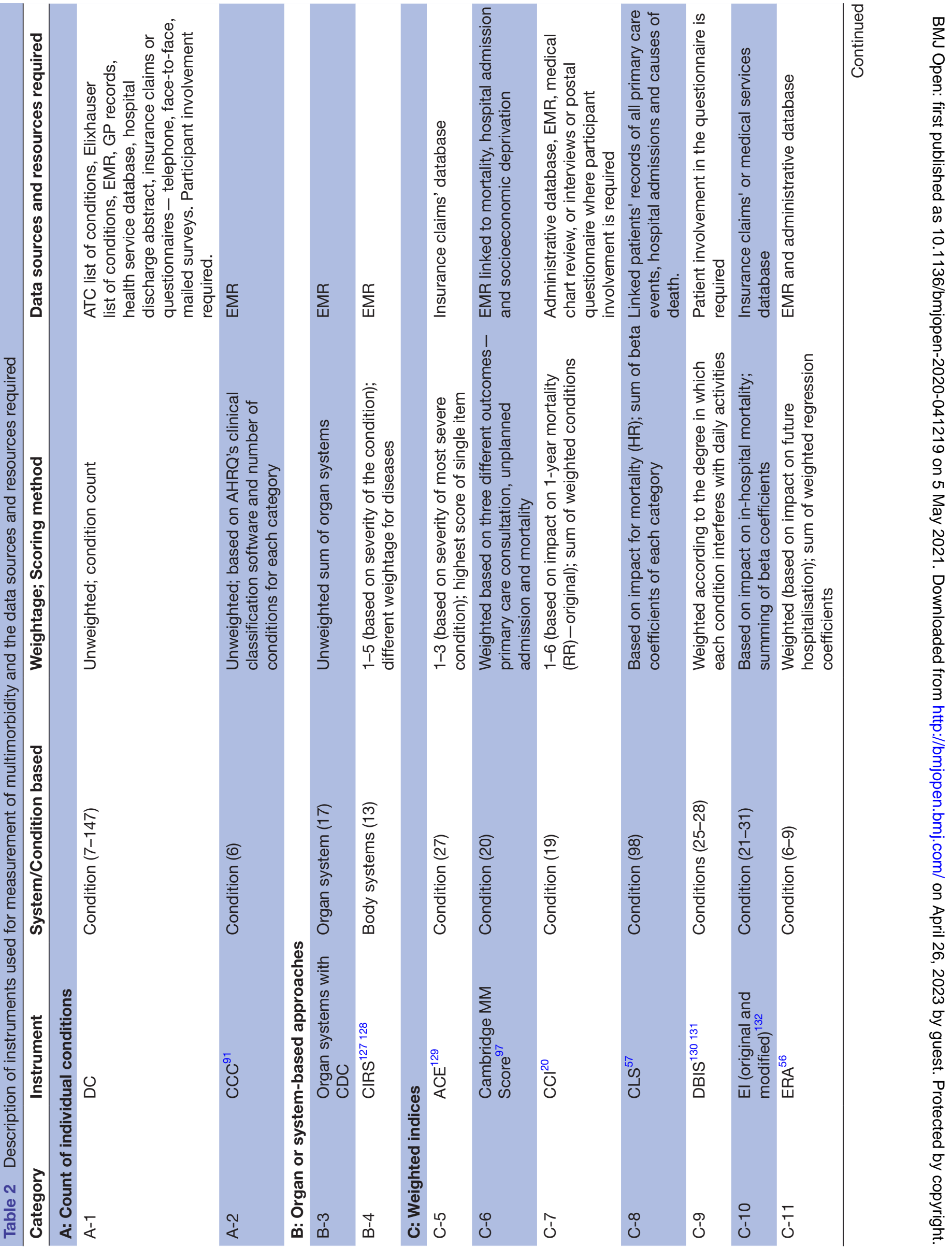




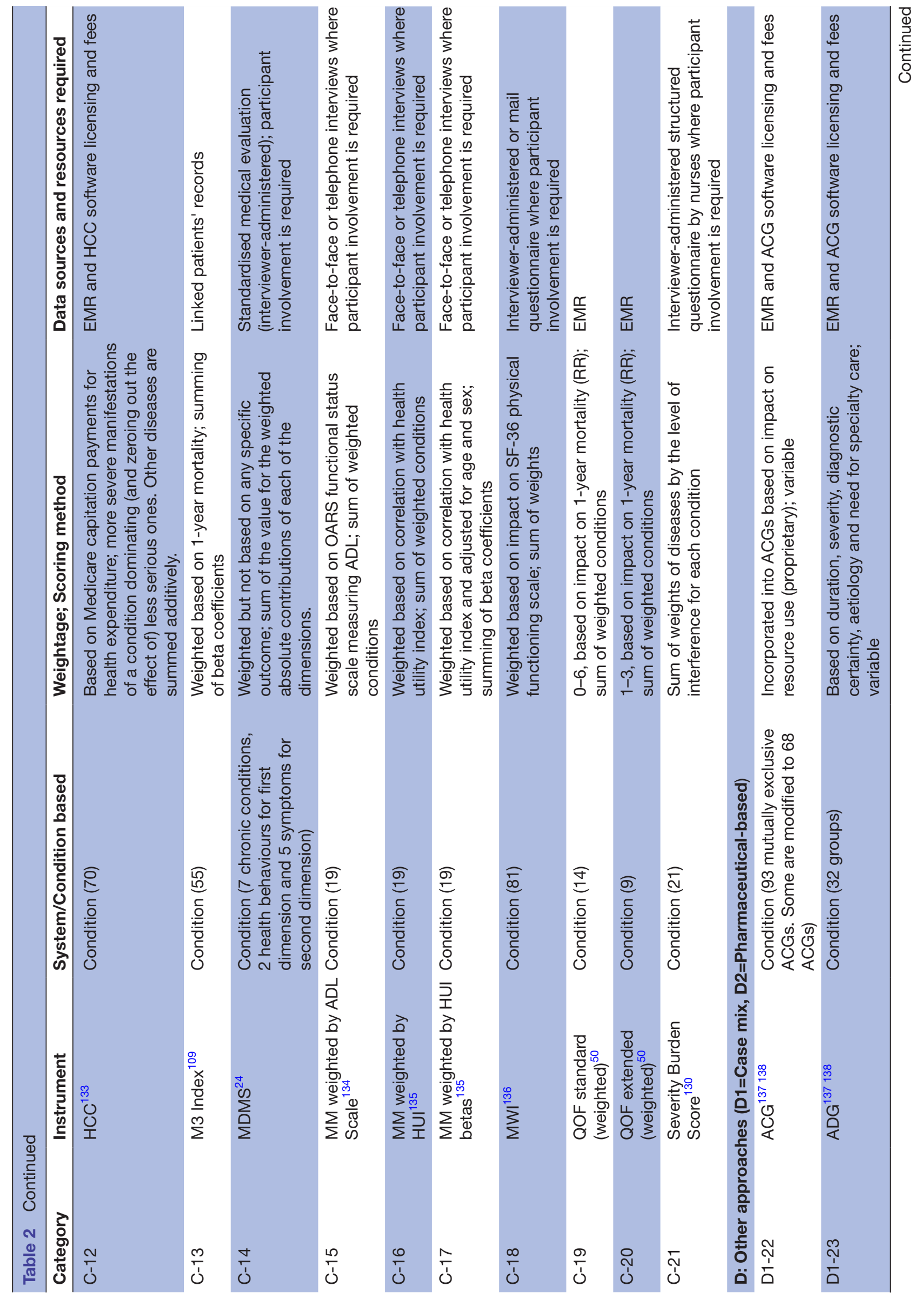

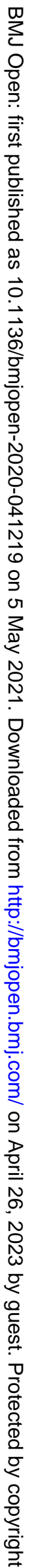




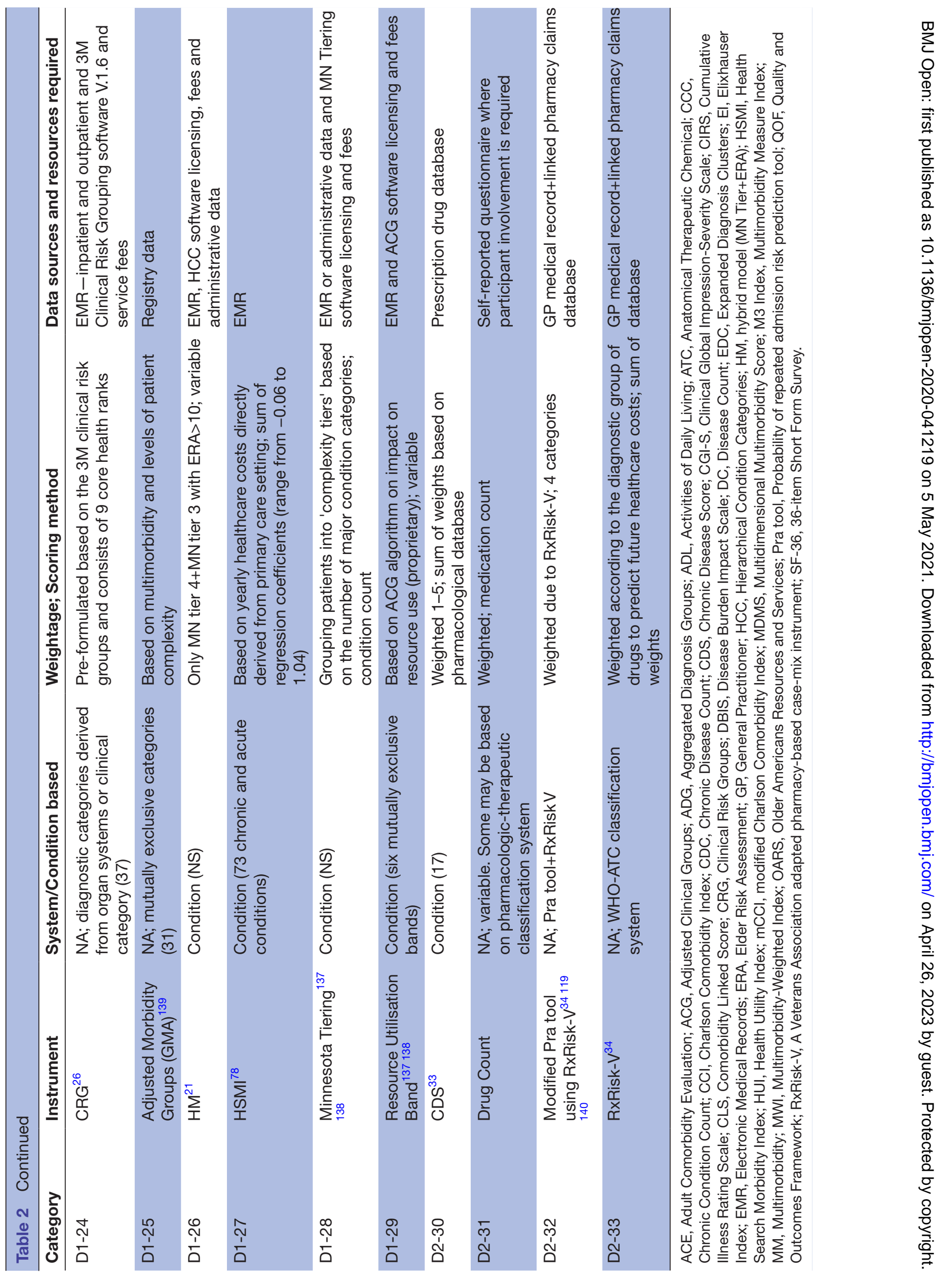


Table 3 Summary of multimorbidity instruments and their associations with outcomes measured from all the included studies

Association between outcomes and multimorbidity

Multimorbidity measures

Evidence of an association

No evidence of an association

\section{$\mathrm{A}=$ Count of individual conditions}

\section{DC (many different groupings ranging} from $7^{6476113}$ to $147^{66}$ conditions and some are further categorised ${ }^{21}$ )
$\mathrm{ADL}$ limitations, ${ }^{43}$ activity limitations, ${ }^{52}$ affective well-being, ${ }^{126}$ cognitive function, ${ }^{82}$ continuity of care (3years), ${ }^{105}$ deficits of perceived general health, ${ }^{104}$ depression, ${ }^{92}$ depressive symptoms, ${ }^{67} 104$ disability, ${ }^{17}$ 658485 emergency hospital admission (2 years), ${ }^{120}$ fallrelated injuries, ${ }^{71}$ fall risk, ${ }^{76}$ frequency of contacts with physicians (1 year), ${ }^{116}$ functional capacity, ${ }^{93}$ functional decline (2 years), ${ }^{120}$ functional Impairment, ${ }^{111}$ functional limitations, ${ }^{74}$ future physical functioning, ${ }^{28}$ general health, ${ }^{52}$ healthcare costs, ${ }^{23} 91$ health-related quality of life, ${ }^{68} 758190115$ hospitalisation (3years), ${ }^{18}$ hospital admissions (1 year), ${ }^{95} 9699$ hospital outpatient visits (1 year), ${ }^{114}$ hospitalisation/emergency department visits, ${ }^{114}$ life satisfaction, ${ }^{22}$ mental distress, ${ }^{52}$ mortality (1 year), ${ }^{99}$ (3 years), ${ }^{1848}$ (5 years), ${ }^{110}$ (10 years), ${ }^{28}$ number of contacts with general practice (1 year), ${ }^{117}$ number of medications prescribed (1 year), ${ }^{117}$ number of mentally unhealthy days, ${ }^{434}$ number of physically unhealthy days, ${ }^{43} 44$ number of different ambulatory physicians contacted (1 year), ${ }^{116}$ number of primary care consultations (1 year), ${ }^{48}$ (3 years), ${ }^{48}$ number of referrals (1 year), ${ }^{117}$ outpatient/Inpatient service use, ${ }^{53}$ physical distress, ${ }^{52}$ physical function, ${ }^{55}$ prescription costs, ${ }^{70}$ perceived health status, ${ }^{22}$ presence of mental health disorder, ${ }^{66}$ primary care consultations (1 year period), ${ }^{105}$ (3years), ${ }^{105}$ primary care experience-self-reported, ${ }^{83}$ primary healthcare cost, ${ }^{47}$ primary healthcare use, ${ }^{89}$ potentially preventable unplanned admission (1-year period), ${ }^{95}$ quality-adjusted life years, ${ }^{72} 73$ quality of life, ${ }^{93} 98101102$ self-rated health, ${ }^{118}$ self-reported falls (12 months), ${ }^{108}$ symptom burden, ${ }^{101}$ self-rated Health, ${ }^{64} 85$ self-perceived health, ${ }^{93}$ total number of consultation, ${ }^{42}$ total health care costs ${ }^{42}$

Healthcare costs, ${ }^{21}$ hospital admissions (1 year), ${ }^{21}$ number of emergency department visits (1 year), ${ }^{21}$ readmission within 30 days $\left(1\right.$ year) ${ }^{21}$

\section{B=Organ or system-based approaches}

Organ systems with CDC

CIRS

C=Weighted indices

ACE

Cambridge MM Score

$\mathrm{CCl}$

\section{Presence of depressive or anxiety disorder ${ }^{19}$}

Disability, ${ }_{17}$ frailty,${ }^{17}$ healthcare utilisation, ${ }^{54}$ hospitalisation Functional decline ${ }^{18}$

(3years), ${ }^{18}$ mortality ${ }^{18}$

Healthcare expenditure ${ }^{23}$

Mortality, ${ }^{97}$ primary care consultation, ${ }^{97}$ unplanned admission

Ambulatory care-sensitive hospitalisations (acute and chronic), ${ }^{106}$ disability, ${ }^{17}$ emergency department visits (1 year), ${ }^{21}$ emergency hospital admission (2 years), ${ }^{119}$ frailty, ${ }^{17}$ functional decline (2 years) ${ }^{119}$ future physical functioning, ${ }^{28}$ healthcare expenditure, ${ }^{21}$ hip fractures, ${ }^{103}$ hospitalisation (1 year), ${ }^{2164} 8099109$ hospitalisation (3years), ${ }^{18}$ mortality (1 year), ${ }^{506399} 109$ mortality (3years), ${ }^{18}$ (5years), ${ }^{51} 63$ (10years), ${ }^{5163}$ (15, 20, 25 years), ${ }^{51}$ number of primary care consultations (3years), ${ }^{48}$ number of primary care physician visits (1 year), ${ }^{107}$ number of specialist visits (1 year), ${ }^{107}$ potentially preventable unplanned admission (1 year), ${ }^{96}$ presence of critical illness, ${ }^{46}$ primary healthcare cost, ${ }^{47}$ mortality (1 year), ${ }^{57} 58$ (3 years), ${ }^{4859}$ (5 years), ${ }^{5860}$

${ }^{62}$ (7 years), ${ }^{86}$ (10years), ${ }^{28}$ readmission within 30 days (1 year) ${ }^{21}$ successful ageing ${ }^{61}$
Functional decline,${ }^{18}$ quality of cardiovascular preventive care,${ }^{27}$ quality of preventive care ${ }^{27}$ 
Table 3 Continued

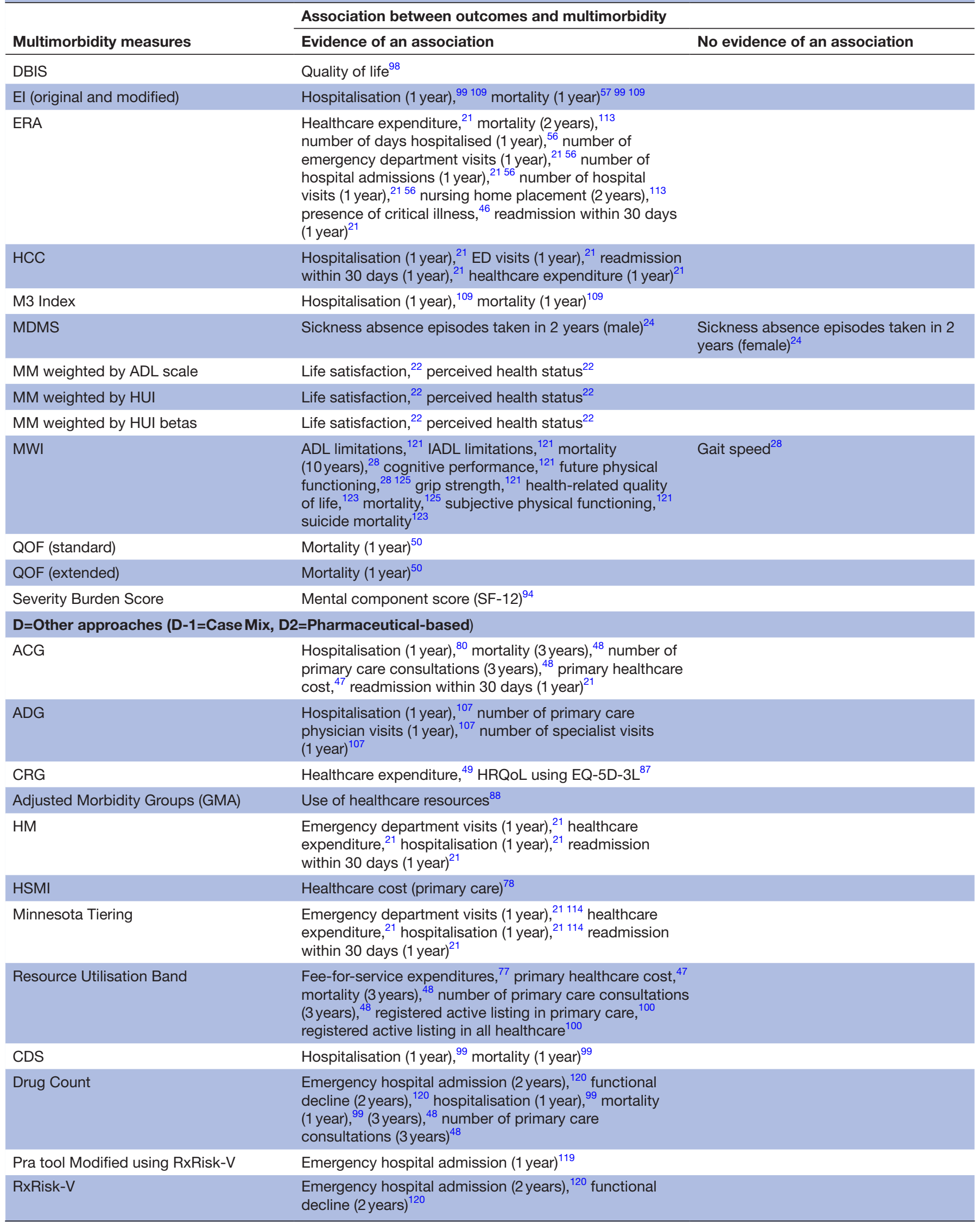


ACE-27, Adult Comorbidity Evaluation; ACG, Adjusted Clinical Groups; ADG, Aggregated Diagnosis Groups; ADL, Activities of Daily Living; CCC, Chronic Condition Count; CCI, Charlson Comorbidity Index; CDC, Chronic Disease Count; CDS, Chronic Disease Score; CIRS, Cumulative Illness Rating Scale; CLS, Comorbidity Linked Score; CRG, Clinical Risk Groups; DBIS, Disease Burden Impact Scale; DC, Disease Count; El, Elixhauser Index; ERA, Elder Risk Assessment; HCC, Hierarchical Condition Categories; HM, Hybrid Model (MN Tier+ERA); HRQoL, health-related quality of life; HSMI, Health Search Morbidity Index; HUI, Health Utility Index; MDMS, Multidimensional Multimorbidity Score; M3 Index, Multimorbidity Measure (M3) Index; MM, Multimorbidity; MWI, Multimorbidity-Weighted Index; Pra tool, Probability of repeated admission risk prediction tool; QOF, Quality and Outcomes Framework; RxRisk-V, A Veterans Association adapted pharmacy-based case-mix instrument; SF-12, Short Form-12.

\section{Organ or system-based approaches}

There were two instruments in this category. They were Cumulative Illness Rating Scale (CIRS) ${ }^{17}$ and Organ Systems with Chronic Disease Count (Organ-CDC). ${ }^{19}$

\section{Weighted indices}

There were 17 unique weighted instruments found in the included studies. The original CCI with its different modifications was the most frequently used instrument and was used in 29 studies. The CCI was based on Disease Count, but the 17 conditions were weighted originally based on their impact on 1-year mortality. ${ }^{20}$ The final score was derived by the summation of all the weighted conditions. There were many variations and modifications of the score including the addition of psychosocial factors. The CCI instrument was found to be associated with multiple outcomes other than 1-year mortality.

Most of the other weighted index instruments were novel, like the Multimorbidity-Weighted Index (MWI), in which the investigators built multivariable prognostic models from a set of potential predictor conditions and weighted the conditions based on an outcome of clinical interest. The most common outcomes chosen were mortality and physical function. Other outcomes included health expenditure, ${ }^{21}$ health utility index ${ }^{22}$ and severity of the most severe condition. ${ }^{23}$ The Multidimensional Multimorbidity Score (MDMS) ${ }^{24}$ was unique as it was weighted based on health behaviours and patient symptoms and not based on any specific outcome.

\section{Other approaches to measuring multimorbidity}

Other approaches included case-mix and pharmaceuticalbased instruments. For case-mix approach, the ACG and Resource Utilisation Band were the most commonly used instruments. ${ }^{25}$ Most of the case-mix instruments required proprietary software licenses from the USA and obtained data from electronic medical records or administrative data. The Clinical Risk Groups instrument was similar but took into account the severity of individual conditions. ${ }^{26}$

The second group of instruments in this category was related to pharmaceutical data. The most frequent type was the unweighted Drug Count. The other three (Chronic Disease Score, A Veterans Association adapted pharmacy-based case-mix instrument like RxRisk-V and modified Probability of repeated admission risk prediction tool using RxRisk-V) were all weighted indices. Except for the Drug Count that was based on a self-report questionnaire, the rest required a prescription drug database to obtain the data.

\section{Outcomes}

We classified the 150 outcomes into 17 categories as reported in the core outcomes set of multimorbidity research (COSmm). ${ }^{10}$ The most commonly reported outcomes were healthcare use $(n=45)$, mortality $(n=18)$, health-related quality of life $(n=18)$ and physical function $(n=13)$. The different studies unanimously showed that higher levels of multimorbidity was associated with higher healthcare use and mortality, lower healthrelated quality of life and poorer physical function. Seven outcomes in the COSmm were not found in all the 96 studies. These were treatment burden, self-management behaviour, self-efficacy, adherence, communications, shared decision-making and prioritisation. There were 19 outcomes that were not described in the COSmm. These included cognitive function, risk of suicide, frailty and falls. The outcomes not found to have any association with the instruments for measuring the level of multimorbidity were preventive care, ${ }^{27}$ sickness absence episodes $(\text { female })^{24}$ and gait speed. ${ }^{28}$

\section{DISCUSSION}

\section{Summary of findings}

Thirty-three unique instruments for measuring the level of multimorbidity were identified and categorised according to the classification by Sarfati. ${ }^{8}$ The most commonly used instrument was 'Disease Count'. It was also the only instrument that was associated with the three essential outcomes from the core outcomes set of multimorbidity research (COSmm) ${ }^{10}$ that is, quality of life, mental health and mortality.

\section{Comparison with previous research}

Although the most common instrument identified in this systematic review was similar to that of Huntley et $a l^{12}$ several instruments including Duke Severity of Illness Checklist (DUSOI) and Functional Comorbidity Index identified in their article were not found in this systematic review. The possible reasons for not finding these instruments in this review could be due to the lack of interest in the instrument by the research community in recent years (to our knowledge, the last publication using DUSOI was 
in 2004), ${ }^{29}$ or the exclusion of studies specifying an index condition.

\section{Advantages and disadvantages of selected instruments Disease Count}

The advantage of using 'Disease Count' is its simplicity and the ease of data ascertainment with minimal resources required. However, using 'Disease Count' does not consider the severity of each condition where the complexity of multimorbidity may not be properly addressed. ${ }^{30}$ The other disadvantage noted was the lack of transparency in the operational definition of multimorbidity, especially regarding the list of conditions considered for multimorbidity and the cut-points used. Despite its simplicity, the level of multimorbidity measured using 'Disease Count' was the only instrument that was found to be associated with the three essential core outcomes (quality of life, mental health and mortality).

\section{Weighted indices}

The common weighted indices identified in this systematic review were CCI, Elders Risk Assessment (ERA), Elixhauser Index (EI) and MWI. These weighted indices were often used in prognostic models to build complex multivariable regression models in which the weights were calculated from hazard ratios, odds ratios or regression coefficients. ${ }^{31}$

The advantage of these weighted indices is that the weights allow the adaptation of an index to a specific outcome. An investigator could recalibrate the correct weight by creating a prognostic model to produce a contextualised instrument for a different setting. Prognostic models can provide clinically relevant risk stratification and help to allocate resources. ${ }^{32}$ The disadvantage of such indices is that calculated weights are greatly influenced by the population, outcomes used, and the instrument's original conception and purpose, hampering the ability to compare across studies.

\section{Case-mix}

The ACG system has a good track record in the USA and several other countries, especially for measuring the outcomes of healthcare utilisation. However, the instrument is proprietary, and the exact algorithm of the instrument is not open to the public and may not be suitable in certain settings. The Clinical Risk Group (CRG) system has a good track record in Spain. It measures the severity of each condition and its algorithm is fully transparent. The common disadvantage of both systems is the financial costs involved in obtaining the license.

\section{Pharmaceutical-based instruments}

Medication-based indices include versions of the Chronic Disease Score, ${ }^{33}$ which later became known as the RxRisk, ${ }^{34}$ and its adaptation for use in the veteran population, the RxRisk-V. ${ }^{35}$ Like the Disease Count, its main advantage is the ease of use with minimal resources required. However, many studies were not transparent regarding which type of drugs were included.

\section{Data sources}

Data sources used by these instruments included medical record information, patient self-report, clinical judgement and large administrative databases. Each data source has its inherent advantages and disadvantages. For patient self-report, patients with cognitive impairment may under-report symptoms and may be seen less frequently by their physicians, resulting in an underrecognition or undertreatment of conditions. ${ }^{36}$ It has also been shown that health administrative data based on billing system underestimated the prevalence of many chronic conditions. ${ }^{37}$

The available data in a particular setting may strongly influence the ultimate instrument chosen for multimorbidity research. As there is currently no consensus on the gold standard for sources of data, it is difficult to assess which data source was superior from this review.

\section{Outcomes}

There were 17 multimorbidity outcomes identified by a Delphi process involving a panel of international experts in multimorbidity intervention studies. ${ }^{10}$ However, only 10 out of the 17 outcomes were reported in the 96 studies identified in this systematic review. The most common outcome that was investigated was healthcare use. The seven missing outcomes belong to "patient-reported impact and behaviours' and 'consultation-related' outcome groups, most likely indicating that there is a dearth of multimorbidity studies looking at these two groups of outcomes measures.

\section{Clinical implications}

Ideally, a single instrument measuring the level of multimorbidity should be able to predict a variety of relevant outcomes. However, Byles et a $\hat{l}^{38}$ reported that a single instrument could not be used to predict different outcomes, in different patient groups and settings, unless different weights were assigned to these factors in calculating a score. Such multiple-scoring instruments may be the way forward for validation of prognostic models for different outcomes and different populations with established multimorbidity instruments. For example, depending on the outcome, study population and setting, the choice of conditions included in the multiple-scoring instrument should include those with a high prevalence in that study population and the weights should be determined by their significant impact (ie, outcome) on the affected population.

For pragmatic reasons, the final selection of the conditions to be included in such a multiple-scoring instrument may still have to take into account the availability of relevant and reliable data. A certain degree of reductionism will also have to be accepted because a single instrument will not be able to encompass all the nuances of the different interactions of chronic conditions on an individual living in his/her unique milieu. We recommend that researchers perform validation studies using the instruments listed in this systematic review to adjust 
the weights according to the specific outcome of interest for the study population relevant to their setting.

\section{Strengths and limitations of the study}

The main strengths of this systematic review were the involvement of a health science librarian in our search strategy, a published protocol, adherence to the protocol without major changes during the systematic review process, ${ }^{39}$ and the critical appraisal of all the primary studies with a risk of bias assessment tool.

The systematic review had several limitations. We excluded grey literature and included only studies that were published in the English language. We also did not contact authors directly for a suggestion of studies, nor identified a list of instruments from the preliminary search and then performed an additional search using the same databases. ${ }^{40}$ Additionally, this systematic review did not review the validity and reliability of all the instruments as it was beyond the scope of the intended work. We have, however, included the references of the original articles or validation studies in table 2 for each of the instrument where available. Finally, this review specifically aimed to look at the association of the level of multimorbidity as the main independent variable and excluded the level of multimorbidity as a mediating, confounding or effect-modifying variable. This strict criterion excluded 17 studies (figure 1) as a result. Excluding these 17 studies did not alter the findings as the instruments used in all the 17 studies were Disease Count $(n=9)$, CIRS $(n=3)$, CCI $(n=3)$, EI $(n=1)$ and Aggregated Diagnosis Groups $(n=1)$ where no new instruments were identified.

\section{CONCLUSIONS}

In this systematic review, we found 33 instruments for measuring the level of multimorbidity in communitydwelling individuals that predict or explore the association of multimorbidity with at least one specified outcome. Disease Count and weighted indices like the CCI, the ERA and EI were commonly used for measuring the level of multimorbidity. Other approaches to measuring the level of multimorbidity included case-mix or pharmaceuticalbased instruments.

We found continuing interest in measuring the level of multimorbidity with Disease Count and Drug Count. There has also been a rise in the development of novel weighted indices using prognostic models or validation of existing well-established instruments like the CCI over the last few years. There is currently an absence of a gold standard for where to obtain chronic disease information. The most suitable instrument will depend on the specified outcome of interest, the study population and the type of data and resources available.

Finally, there is still much work to improve on the body of knowledge of multimorbidity when most investigators in the last decade measured multimorbidity without including some of the important outcome measures of multimorbidity. We also suggest that a clear description of the instruments is required in the publication of multimorbidity studies to counter the frequent lack of information currently seen so as to contribute to robust multimorbidity research in future.

\section{Author affiliations}

${ }^{1}$ Clinical Research Unit, National Healthcare Group Polyclinics, Singapore ${ }^{2}$ Lee Kong Chian School of Medicine, Nanyang Technological University, Singapore

${ }^{3}$ Saw Swee Hock School of Public Health, National University of Singapore, Singapore

${ }^{4}$ Department of Epidemiology and Biostatistics, Western University Schulich School of Medicine and Dentistry, London, Ontario, Canada

${ }^{5}$ Centre for Studies in Family Medicine, Department of Family Medicine, Western University Schulich School of Medicine and Dentistry, London, Ontario, Canada

${ }^{6}$ Department of Family Medicine and Emergency Medicine, Université de Sherbrooke, Sherbrooke, Quebec, Canada

Acknowledgements We would like to thank Mr John Costella, Collections and Content Strategies Librarian, Western University for his assistance and guidance in the search strategy. We would also like to thank Dr Mohammed Zulhakim, Family Medicine Resident Doctor, Ministry of Health Holdings, for his help in testing out the various risk assessment tools and his suggestions on the modified NewcastleOttawa Scale.

Contributors All authors made substantial contribution to the design of the work. $\mathrm{BLR}, \mathrm{MF}$ and MS mentored and provided guidance to the review. ESL, together with a health science librarian, created the search strategy and conducted the search. ESL, EH, TSH, HLK and FYW screened articles, extracted and analysed data. ESL drafted the manuscript with valuable inputs from BLR, MF and MS and all authors reviewed drafts and approved the final version.

Funding This study was supported by the Singapore Ministry of Health's National Medical Research Council under the Centre Grant Programme (Ref No: NMRC/CG/ C019/2017).

Competing interests None declared.

Patient consent for publication Not required.

Provenance and peer review Not commissioned; externally peer reviewed.

Data availability statement Data sharing not applicable as no datasets generated and/or analysed for this study. All data relevant to the study are included in the article or uploaded as supplemental information. There are no additional, unpublished data arising from this research.

Supplemental material This content has been supplied by the author(s). It has not been vetted by BMJ Publishing Group Limited (BMJ) and may not have been peer-reviewed. Any opinions or recommendations discussed are solely those of the author(s) and are not endorsed by BMJ. BMJ disclaims all liability and responsibility arising from any reliance placed on the content. Where the content includes any translated material, BMJ does not warrant the accuracy and reliability of the translations (including but not limited to local regulations, clinical guidelines, terminology, drug names and drug dosages), and is not responsible for any error and/or omissions arising from translation and adaptation or otherwise.

Open access This is an open access article distributed in accordance with the Creative Commons Attribution Non Commercial (CC BY-NC 4.0) license, which permits others to distribute, remix, adapt, build upon this work non-commercially, and license their derivative works on different terms, provided the original work is properly cited, appropriate credit is given, any changes made indicated, and the use is non-commercial. See: http://creativecommons.org/licenses/by-nc/4.0/.

ORCID iD

Eng Sing Lee http://orcid.org/0000-0003-4963-535X

\section{REFERENCES}

1 WHO. The World Health Report 2008. Primary Care - Now more than ever, 2008.

2 Huber M, Knottnerus JA, Green L, et al. How should we define health? BMJ 2011;343:d4163.

3 Coventry PA, Small N, Panagioti M, et al. Living with complexity; marshalling resources: a systematic review and qualitative meta- 
synthesis of lived experience of mental and physical multimorbidity. BMC Fam Pract 2015:16:171.

4 Liddy C, Blazkho V, Mill K. Challenges of self-management when living with multiple chronic conditions: systematic review of the qualitative literature. Can Fam Physician 2014;60:1123-33.

5 Lugtenberg M, Burgers JS, Clancy C, et al. Current guidelines have limited applicability to patients with comorbid conditions: a systematic analysis of evidence-based guidelines. PLoS One 2011;6:e25987.

6 Tinetti ME, Fried T. The end of the disease era. Am J Med 2004; $116: 179-85$

7 Fortin M, Stewart M, Poitras M-E, et al. A systematic review of prevalence studies on multimorbidity: toward a more uniform methodology. Ann Fam Med 2012;10:142-51.

8 Sarfati D. Review of methods used to measure comorbidity in cancer populations: no gold standard exists. J Clin Epidemiol 2012:65:924-33.

9 Sarfati D. How Do We Measure Comorbidity? In: Koczwara B, ed. Cancer and Chronic Conditions - Addressing the problem of multimorbidity in cancer patients and survivors. Springer, 2016: 35-70.

10 Smith SM, Wallace E, Salisbury C, et al. A core outcome set for multimorbidity research (COSmm). Ann Fam Med 2018;16:132-8.

11 Kirkham JJ, Gorst S, Altman DG, et al. Core outcome SetSTAndards for reporting: the COS-STAR statement. PLoS Med 2016;13:e1002148.

12 Huntley AL, Johnson R, Purdy S, et al. Measures of multimorbidity and morbidity burden for use in primary care and community settings: a systematic review and guide. Ann Fam Med 2012:10:134-41.

13 Lee ES, EQ H, Koh HL. A systematic review on the instruments used for measuring the severity of multimorbidity, 2018. Available: https://www.crd.york.ac.uk/prospero/display_record.php? RecordID $=105297$

14 van den Akker M, Buntinx F, Metsemakers JF, et al. Multimorbidity in general practice: prevalence, incidence, and determinants of co-occurring chronic and recurrent diseases. J Clin Epidemiol 1998:51:367-75.

15 Alshabanat A, Zafari Z, Albanyan O, et al. Asthma and COPD overlap syndrome (ACOs): a systematic review and meta analysis. PLoS One 2015;10:e0136065.

16 Wells GA, Shhea B, O'Connell D. The Newcastle-Ottawa scale (NOS) for assessing the quality of nonrandomised studies in meta-analyses. Available: http://www.ohri.ca/programs/clinical_ epidemiology/oxford.asp [Accessed 6 Apr 2018].

17 Boeckxstaens P, Vaes B, Legrand D, et al. The relationship of multimorbidity with disability and frailty in the oldest patients: a cross-sectional analysis of three measures of multimorbidity in the BELFRAIL cohort. Eur J Gen Pract 2015;21:39-44.

18 Boeckxstaens P, Vaes B, Van Pottelbergh G, et al. Multimorbidity measures were poor predictors of adverse events in patients aged $\geq 80$ years: a prospective cohort study. J Clin Epidemiol 2015;68:220-7.

19 DiNapoli EA, Bramoweth AD, Whiteman KL, et al. Mood disorders in middle-aged and older veterans with multimorbidity. J Aging Health 2017;29:657-68.

20 Charlson ME, Pompei P, Ales KL, et al. A new method of classifying prognostic comorbidity in longitudinal studies: development and validation. J Chronic Dis 1987:40:373-83.

21 Haas LR, Takahashi PY, Shah ND, et al. Risk-Stratification methods for identifying patients for care coordination. Am J Manag Care 2013;19:725-32.

22 Wister AV, Levasseur M, Griffith LE, et al. Estimating multiple morbidity disease burden among older persons: a convergent construct validity study to discriminate among six chronic illness measures, CCHS 2008/09. BMC Geriatr 2015;15:12

23 Hwang W, LaClair M, Camacho F, et al. Persistent high utilization in a privately insured population. Am J Manag Care 2015;21:309-16.

24 Ubalde-Lopez M, Delclos GL, Benavides FG, et al. Measuring multimorbidity in a working population: the effect on incident sickness absence. Int Arch Occup Environ Health 2016;89:667-78.

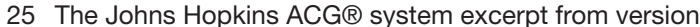
11.0 technical reference guide 2014. Available: https://www. healthpartners.com/ucm/groups/public/@hp/@public/documents/ documents/cntrb 035024.pdf [Accessed 10 Sep 2019].

26 Hughes JS, Averill RF, Eisenhandler J, et al. Clinical risk groups (CRGs): a classification system for risk-adjusted capitation-based payment and health care management. Med Care 2004;42:81-90.

27 Streit S, da Costa BR, Bauer DC, et al. Multimorbidity and quality of preventive care in Swiss university primary care cohorts. PLoS One 2014;9:e96142.
28 Wei MY, Mukamal KJ. Multimorbidity, mortality, and long-term physical functioning in 3 prospective cohorts of communitydwelling adults. Am J Epidemiol 2018;187:103-12.

29 Moll van Charante E, Hartman E, Yzermans J, et al. The first general practitioner hospital in the Netherlands: towards a new form of integrated care? Scand J Prim Health Care 2004;22:38-43.

30 Salive ME. Multimorbidity in older adults. Epidemiol Rev 2013;35:75-83.

31 Moons KGM, Royston P, Vergouwe Y, et al. Prognosis and prognostic research: what, why, and how? BMJ 2009;338:b375.

32 Alonso-Morán E, Nuño-Solinis R, Onder G, et al. Multimorbidity in risk stratification tools to predict negative outcomes in adult population. Eur J Intern Med 2015;26:182-9.

33 Von Korff M, Wagner EH, Saunders K. A chronic disease score from automated pharmacy data. J Clin Epidemiol 1992;45:197-203.

34 Fishman PA, Goodman MJ, Hornbrook MC, et al. Risk adjustment using automated ambulatory pharmacy data: the RxRisk model. Med Care 2003;41:84-99.

35 Sloan KL, Sales AE, Liu C-F, et al. Construction and characteristics of the RxRisk-V: a VA-adapted pharmacy-based case-mix instrument. Med Care 2003;41:761-74.

36 Lash TL, Mor V, Wieland D, et al. Methodology, design, and analytic techniques to address measurement of comorbid disease. $J$ Gerontol A Biol Sci Med Sci 2007;62:281-5.

37 Fortin M, Haggerty J, Sanche S, et al. Self-reported versus health administrative data: implications for assessing chronic illness burden in populations. A cross-sectional study. CMAJ Open 2017;5:E729-33.

38 Byles JE, D'Este C, Parkinson L, et al. Single index of multimorbidity did not predict multiple outcomes. J Clin Epidemiol 2005;58:997-1005

39 Kirkham JJ, Altman DG, Williamson PR. Bias due to changes in specified outcomes during the systematic review process. PLoS One 2010;5:e9810.

40 Yurkovich M, Avina-Zubieta JA, Thomas J, et al. A systematic review identifies valid comorbidity indices derived from administrative health data. J Clin Epidemiol 2015;68:3-14.

41 Agborsangaya CB, Lau D, Lahtinen M, et al. Health-Related quality of life and healthcare utilization in multimorbidity: results of a crosssectional survey. Qual Life Res 2013;22:791-9.

42 Bähler C, Huber CA, Brüngger B, et al. Multimorbidity, health care utilization and costs in an elderly community-dwelling population: a claims data based observational study. BMC Health Serv Res 2015;15:23.

43 Barile JP, Thompson WW, Zack MM, et al. Multiple chronic medical conditions and health-related quality of life in older adults, 2004-2006. Prev Chronic Dis 2013;10:E162.

44 Barile JP, Thompson WW, Zack MM, et al. Activities of daily living, chronic medical conditions, and health-related quality of life in older adults. J Ambul Care Manage 2012;35:292-303.

45 Barnett K, Mercer SW, Norbury M, et al. Epidemiology of multimorbidity and implications for health care, research, and medical education: a cross-sectional study. Lancet 2012;380:37-43.

46 Biehl M, Takahashi PY, Cha SS, et al. Prediction of critical illness in elderly outpatients using elder risk assessment: a population-based study. Clin Interv Aging 2016;11:829-34.

47 Brilleman SL, Gravelle H, Hollinghurst S, et al. Keep it simple? Predicting primary health care costs with clinical morbidity measures. J Health Econ 2014;35:109-22.

48 Brilleman SL, Salisbury C. Comparing measures of multimorbidity to predict outcomes in primary care: a cross sectional study. Fam Pract 2013;30:172-8.

49 Caballer-Tarazona V, Guadalajara-Olmeda N, Vivas-Consuelo D. Predicting healthcare expenditure by multimorbidity groups. Health Policy 2019;123:427-34.

50 Carey IM, Shah SM, Harris T, et al. A new simple primary care morbidity score predicted mortality and better explains between practice variations than the Charlson index. J Clin Epidemio 2013;66:436-44.

51 Chapman BP, Weiss A, Fiscella K, et al. Mortality risk prediction: can comorbidity indices be improved with psychosocial data? Med Care 2015;53:909-15.

52 Chen H-Y, Baumgardner DJ, Rice JP. Health-Related quality of life among adults with multiple chronic conditions in the United States, behavioral risk factor surveillance system, 2007. Prev Chronic Dis 2011;8:A09.

53 Chen $\mathrm{H}$, Cheng M, Zhuang Y, et al. Multimorbidity among middleaged and older persons in urban China: prevalence, characteristics and health service utilization. Geriatr Gerontol Int 2018;18:1447-52.

54 Chu T, Lau P, Cheng RSY. A study on the prevalence of multi morbidities of diseases and utilisation of public healthcare services 
in the New Territories West area of Hong Kong. The Hong Kong Practitioner 2018;40.

55 Clynes MA, Bevilacqua G, Jameson KA, et al. Does self-report of multimorbidity in later life predict impaired physical functioning, and might this be useful in clinical practice? Aging Clin Exp Res 2020;32:1443-50.

56 Crane SJ, Tung EE, Hanson GJ, et al. Use of an electronic administrative database to identify older community dwelling adults at high-risk for hospitalization or emergency department visits: the elders risk assessment index. BMC Health Serv Res 2010;10:338.

57 Crooks CJ, Card TR, West J. The use of a Bayesian hierarchy to develop and validate a co-morbidity score to predict mortality for linked primary and secondary care data from the NHS in England. PLoS One 2016;11:e0165507.

58 Crooks CJ, West J, Card TR. A comparison of the recording of comorbidity in primary and secondary care by using the Charlson index to predict short-term and long-term survival in a routine linked data cohort. BMJ Open 2015;5:e007974.

59 Formiga F, Ferrer A, Chivite D, et al. Utility of geriatric assessment to predict mortality in the oldest old: the Octabaix study 3-year follow-up. Rejuvenation Res 2013;16:279-84.

60 Formiga F, Ferrer A, Chivite D, et al. Predictors of long-term survival in nonagenarians: the NonaSantfeliu study. Age Ageing 2011;40:111-6.

61 Formiga F, Ferrer A, Megido MJ, et al. Low co-morbidity, low levels of malnutrition, and low risk of falls in a community-dwelling sample of 85-year-olds are associated with successful aging: the Octabaix study. Rejuvenation Res 2011;14:309-14.

62 Formiga F, Ferrer A, Padros G, et al. Evidence of functional declining and global comorbidity measured at baseline proved to be the strongest predictors for long-term death in elderly community residents aged 85 years: a 5 -year follow-up evaluation, the OCTABAIX study. Clin Interv Aging 2016;11:437-44.

63 Fraccaro $\mathrm{P}$, Kontopantelis $\mathrm{E}$, Sperrin $\mathrm{M}$, et al. Predicting mortality from change-over-time in the Charlson comorbidity index: a retrospective cohort study in a data-intensive UK health system. Medicine 2016;95:e4973.

64 Galenkamp H, Braam AW, Huisman M, et al. Somatic multimorbidity and self-rated health in the older population. J Gerontol B Psychol Sci Soc Sci 2011:66:380-6.

65 Garin N, Olaya B, Moneta MV, et al. Impact of multimorbidity on disability and quality of life in the Spanish older population. PLOS One 2014;9:e111498.

66 Glynn LG, Valderas JM, Healy P, et al. The prevalence of multimorbidity in primary care and its effect on health care utilization and cost. Fam Pract 2011;28:516-23.

67 Gunn JM, Ayton DR, Densley K, et al. The association between chronic illness, multimorbidity and depressive symptoms in an Australian primary care cohort. Soc Psychiatry Psychiatr Epidemiol 2012:47:175-84.

68 Hanmer J, Vanness D, Gangnon R, et al. Three methods tested to model SF-6D health utilities for health states involving comorbidity/ co-occurring conditions. J Clin Epidemiol 2010;63:331-41.

$69 \mathrm{Hu} \mathrm{T}$, Dattani ND, Cox KA, et al. Effect of comorbidities and medications on frequency of primary care visits among older patients. Can Fam Physician 2017:63:45-50.

70 Isaacs AA, Manga N, Le Grange C, et al. A snapshot of noncommunicable disease profiles and their prescription costs at ten primary healthcare facilities in the in the Western half of the Cape town Metropole. South African Family Practice 2014;56:43-9.

71 Jennings LA, Reuben DB, Kim S-B, et al. Targeting a high-risk group for fall prevention: strategies for health plans. Am J Manag Care 2015;21:e519-26.

72 Jia H, Lubetkin El, Barile JP, et al. Quality-Adjusted life years (QALY) for 15 chronic conditions and combinations of conditions among US adults aged 65 and older. Med Care 2018;56:740-6.

$73 \mathrm{Jia} \mathrm{H}$, Lubetkin El. Impact of nine chronic conditions for US adults aged 65 years and older: an application of a hybrid estimator of quality-adjusted life years throughout remainder of lifetime. Qual Life Res 2016;25:1921-9.

74 Jindai K, Nielson CM, Vorderstrasse BA, et al. Multimorbidity and functional limitations among adults 65 or older, NHANES 20052012. Prev Chronic Dis 2016;13:E151.

$75 \mathrm{Kim} \mathrm{K}-\mathrm{I}$, Lee JH, Kim C-H. Impaired health-related quality of life in elderly women is associated with multimorbidity: results from the Korean National health and nutrition examination survey. Gend Med 2012;9:309-18.

76 Kojima T, Akishita M, Nakamura T, et al. Association of polypharmacy with fall risk among geriatric outpatients. Geriatr Gerontol Int 2011;11:438-44.
77 Kristensen T, Olsen KR, Schroll H, et al. Association between feefor-service expenditures and morbidity burden in primary care. Eur $J$ Health Econ 2014;15:599-610.

78 Lapi F, Bianchini E, Cricelli I, et al. Development and validation of a score for adjusting health care costs in general practice. Value Health 2015;18:884-95.

79 Lawson KD, Mercer SW, Wyke S, et al. Double trouble: the impact of multimorbidity and deprivation on preference-weighted health related quality of life a cross sectional analysis of the Scottish health survey. Int J Equity Health 2013;12:67.

80 Lemke KW, Weiner JP, Clark JM. Development and validation of a model for predicting inpatient hospitalization. Med Care 2012;50:131-9.

81 Li J, Green M, Kearns B, et al. Patterns of multimorbidity and their association with health outcomes within Yorkshire, England: baseline results from the Yorkshire health study. BMC Public Health 2016;16:649-49.

82 Loprinzi PD. Multimorbidity, cognitive function, and physical activity. Age 2016;38:8.

83 Macinko J, Andrade FCD, Nunes BP, et al. Primary care and multimorbidity in six Latin American and Caribbean countries. Rev Panam Salud Publica 2019;43:e8:1-9.

84 Marengoni A, Angleman S, Fratiglioni L. Prevalence of disability according to multimorbidity and disease clustering: a populationbased study. J Comorb 2011;1:11-18.

85 McDaid O, Hanly MJ, Richardson K, et al. The effect of multiple chronic conditions on self-rated health, disability and quality of life among the older populations of Northern Ireland and the Republic of ireland: a comparison of two nationally representative crosssectional surveys. BMJ Open 2013;3:e002571.

86 Md Yusof MY, Horan MA, Jones M, et al. Developing a self-reported comorbidity index to predict mortality of community-dwelling older adults. Arch Gerontol Geriatr 2010;50:e63-7.

87 Millá-Perseguer M, Guadalajara-Olmeda N, Vivas-Consuelo D, et al Measurement of health-related quality by multimorbidity groups in primary health care. Health Qual Life Outcomes 2019;17:8.

88 Monterde D, Vela E, Clèries M, et al. Multimorbidity as a predictor of health service utilization in primary care: a registry-based study of the Catalan population. BMC Fam Pract 2020;21:39.

89 Muggah E, Graves E, Bennett C, et al. The impact of multiple chronic diseases on ambulatory care use; a population based study in Ontario, Canada. BMC Health Serv Res 2012;12:452-52.

90 Mujica-Mota RE, Roberts M, Abel G, et al. Common patterns of morbidity and multi-morbidity and their impact on health-related quality of life: evidence from a national survey. Qual Life Res 2015;24:909-18.

91 Naessens JM, Stroebel RJ, Finnie DM, et al. Effect of multiple chronic conditions among working-age adults. Am J Manag Care 2011:17:118-22.

92 Østergaard SD, Foldager L. The association between physical illness and major depressive episode in general practice. Acta Psychiatr Scand 2011;123:290-6.

93 Palladino R, Pennino F, Finbarr M, et al. Multimorbidity and health outcomes in older adults in ten European health systems, 2006-15. Health Aff 2019;38:613-23.

94 Pati S, Swain S, Knottnerus JA, et al. Health related quality of life in multimorbidity: a primary-care based study from Odisha, India. Health Qual Life Outcomes 2019;17:116.

95 Payne RA, Abel GA, Guthrie B, et al. The effect of physical multimorbidity, mental health conditions and socioeconomic deprivation on unplanned admissions to hospital: a retrospective cohort study. CMAJ 2013;185:E221-8.

96 Payne RA, Abel GA, Avery AJ, et al. Is polypharmacy always hazardous? A retrospective cohort analysis using linked electronic health records from primary and secondary care. Br J Clin Pharmacol 2014;77:1073-82.

97 Payne RA, Mendonca SC, Elliott MN, et al. Development and validation of the Cambridge multimorbidity score. CMAJ 2020;192:E107-14.

98 Peters M, Kelly L, Potter CM, et al. Quality of life and burden of morbidity in primary care users with multimorbidity. Patient Relat Outcome Meas 2018;9:103-13.

99 Quail JM, Lix LM, Osman BA, et al. Comparing comorbidity measures for predicting mortality and hospitalization in three population-based cohorts. BMC Health Serv Res 2011;11:146.

100 Ranstad K, Midlöv P, Halling A. Importance of healthcare utilization and multimorbidity level in choosing a primary care provider in Sweden. Scand J Prim Health Care 2014;32:99-105.

101 Reinke LF, Vig EK, Tartaglione EV, et al. Symptom burden and palliative care needs among high-risk veterans with multimorbidity. J Pain Symptom Manage 2019;57:880-9. 
102 Renne I, Gobbens RJ. Effects of frailty and chronic diseases on quality of life in Dutch community-dwelling older adults: a crosssectional study. Clin Interv Aging 2018;13:325-34.

103 Reyes C, Estrada P, Nogués X, et al. The impact of common comorbidities (as measured using the Charlson index) on hip fracture risk in elderly men: a population-based cohort study. Osteoporos Int 2014;25:1751-8. [Erratum appears in Osteoporos Int. 2014 Sep;25(9):2333 Note: Macias, J G [corrected to Gonzalez-Macias, J]].

104 Ryu E, Takahashi PY, Olson JE, et al. Quantifying the importance of disease burden on perceived general health and depressive symptoms in patients within the Mayo clinic Biobank. Health Qual Life Outcomes 2015;13:95.

105 Salisbury C, Johnson L, Purdy S, et al. Epidemiology and impact of multimorbidity in primary care: a retrospective cohort study. $\mathrm{Br} J$ Gen Pract 2011;61:e12-21.

106 Saver BG, Wang C-Y, Dobie SA, et al. The central role of comorbidity in predicting ambulatory care sensitive hospitalizations*. Eur J Public Health 2014;24:66-72.

107 Shadmi E, Balicer RD, Kinder K, et al. Assessing socioeconomic health care utilization inequity in Israel: impact of alternative approaches to morbidity adjustment. BMC Public Health 2011;11:609.

108 Sibley KM, Voth J, Munce SE, et al. Chronic disease and falls in community-dwelling Canadians over 65 years old: a populationbased study exploring associations with number and pattern of chronic conditions. BMC Geriatr 2014;14:22.

109 Stanley J, Sarfati D. The new measuring multimorbidity index predicted mortality better than Charlson and Elixhauser indices among the general population. J Clin Epidemiol 2017;92:99-110.

110 St John PD, Tyas SL, Menec V, et al. Multimorbidity, disability, and mortality in community-dwelling older adults. Can Fam Physician 2014;60:e272-80.

111 St John PD, Tyas SL, Menec V, et al. Multimorbidity predicts functional decline in community-dwelling older adults: prospective cohort study. Can Fam Physician 2019;65:e56-63.

112 Sullivan PW, Ghushchyan VH, Bayliss EA. The impact of comorbidity burden on preference-based health-related quality of life in the United States. Pharmacoeconomics 2012;30:431-42.

113 Takahashi PY, Tung EE, Crane SJ, et al. Use of the elderly risk assessment (era) index to predict 2-year mortality and nursing home placement among community dwelling older adults. Arch Gerontol Geriatr 2012;54:34-8.

114 Takahashi PY, Heien HC, Sangaralingham LR, et al. Enhanced risk prediction model for emergency department use and hospitalizations in patients in a primary care medical home. Am J Manag Care 2016;22:475-83.

115 Tyack Z, Frakes K-A, Barnett A, et al. Predictors of healthrelated quality of life in people with a complex chronic disease including multimorbidity: a longitudinal cohort study. Qual Life Res 2016;25:2579-92

116 van den Bussche $\mathrm{H}$, Schön G, Kolonko T, et al. Patterns of ambulatory medical care utilization in elderly patients with special reference to chronic diseases and multimorbidity--results from a claims data based observational study in Germany. BMC Geriatr 2011:11:54.

117 van Oostrom SH, Picavet HSJ, de Bruin SR, et al. Multimorbidity of chronic diseases and health care utilization in general practice. BMC Fam Pract 2014;15:61.

118 Vos HMM, Bor HH, Rangelrooij-Minkels MJAvan, et al. Multimorbidity in older women: the negative impact of specific combinations of chronic conditions on self-rated health. Eur J Gen Pract 2013;19:117-22.

119 Wallace E, McDowell R, Bennett K, et al. External validation of the probability of repeated admission (PRA) risk prediction tool in older community-dwelling people attending general practice: a prospective cohort study. BMJ Open 2016;6:e012336.
120 Wallace E, McDowell R, Bennett K, et al. Comparison of countbased multimorbidity measures in predicting emergency admission and functional decline in older community-dwelling adults: a prospective cohort study. BMJ Open 2016;6:e013089.

121 Wei MY, Kabeto MU, Langa KM, et al. Multimorbidity and physical and cognitive function: performance of a new MultimorbidityWeighted index. J Gerontol A Biol Sci Med Sci 2018;73:225-32.

122 Wei MY, Kabeto MU, Galecki AT, et al. Physical functioning decline and mortality in older adults with multimorbidity: joint modeling of longitudinal and survival data. J Gerontol A Biol Sci Med Sci 2019;74:226-32.

123 Wei MY, Mukamal KJ. Multimorbidity and mental health-related quality of life and risk of completed suicide. J Am Geriatr Soc 2019;67:511-9.

124 Wei MY, Levine DA, Zahodne LB, et al. Multimorbidity and cognitive decline over 14 years in older Americans. J Gerontol A Biol Sci Med Sci 2020;75:1206-13.

125 Wei MY, Ratz D, Mukamal KJ. Multimorbidity in Medicare beneficiaries: performance of an ICD-Coded MultimorbidityWeighted index. J Am Geriatr Soc 2020;68:999-1006.

126 Wikman A, Wardle J, Steptoe A. Quality of life and affective well-being in middle-aged and older people with chronic medical illnesses: a cross-sectional population based study. PLoS One 2011;6:e18952.

127 Linn BS, Linn MW, Gurel L. Cumulative illness rating scale. J Am Geriatr Soc 1968;16:622-6.

128 Miller MD, Paradis CF, Houck PR, et al. Rating chronic medical illness burden in geropsychiatric practice and research: application of the cumulative illness rating scale. Psychiatry Res 1992;41:237-48.

129 Fleming ST, Sabatino SA, Kimmick G, et al. Developing a claimbased version of the ACE-27 comorbidity index: a comparison with medical record review. Med Care 2011;49:752-60.

130130 Bayliss EA, Ellis JL, Steiner JF. Subjective assessments of comorbidity correlate with quality of life health outcomes: initial validation of a comorbidity assessment instrument. Health Qual Life Outcomes 2005;3:51.

131 Bayliss EA, Ellis JL, Steiner JF. Seniors' self-reported multimorbidity captured biopsychosocial factors not incorporated into two other data-based morbidity measures. J Clin Epidemiol 2009;62:550-7.

132 Elixhauser A, Steiner C, Harris DR, et al. Comorbidity measures for use with administrative data. Med Care 1998;36:8-27.

133 Pope GC, Kautter J, Ellis RP, et al. Risk adjustment of Medicare capitation payments using the CMS-HCC model. Health Care Financ Rev 2004;25:119-41.

134 Fillenbaum GG, Smyer MA. The development, validity, and reliability of the OARS multidimensional functional assessment questionnaire. $J$ Gerontol 1981;36:428-34.

135 Feeny D, Furlong W, Torrance GW, et al. Multiattribute and singleattribute utility functions for the health Utilities index mark 3 system. Med Care 2002;40:113-28.

136 Wei MY, Kawachi I, Okereke Ol, et al. Diverse cumulative impact of chronic diseases on physical health-related quality of life: implications for a measure of multimorbidity. Am J Epidemiol 2016;184:357-65.

137 Starfield B, Weiner J, Mumford L, et al. Ambulatory care groups: a categorization of diagnoses for research and management. Health Serv Res 1991;26:53-74.

138 Weiner JP, Starfield BH, Steinwachs DM, et al. Development and application of a population-oriented measure of ambulatory care case-mix. Med Care 1991;29:452-72.

139 Monterde D, Vela E, Clèries M, et al. [Adjusted morbidity groups: A new multiple morbidity measurement of use in Primary Care]. Aten Primaria 2016;48:674.

140 Boult C, Dowd B, McCaffrey D, et al. Screening elders for risk of hospital admission. J Am Geriatr Soc 1993;41:811-7. 